\title{
A CDRX-based Material Model for Hot Deformation of Aluminium Alloys
}

\author{
Yibo $\mathrm{Li}^{1}$, Bin $\mathrm{Gu}^{2}$, Shuai Jiang ${ }^{3}$, Yaoqiong $\mathrm{Liu}^{2}$, Zhusheng $\mathrm{Shi}^{3}, *$, Jianguo $\mathrm{Lin}^{3}$ \\ ${ }^{1}$ Light Alloy Research Institute, Central South University, Changsha 410083, China \\ ${ }^{2}$ AVIC the First Aircraft Institute, Xi'an 710089, China \\ ${ }^{3}$ Department of Mechanical Engineering, Imperial College London, London SW7 2AZ, UK \\ *Corresponding author: zhusheng.shi@imperial.ac.uk (Z. Shi)
}

\begin{abstract}
During hot deformation, continuous dynamic recrystallisation (CDRX) is believed to occur, and even dominates microstructural evolution in many metallic materials with high stacking fault energy, such as aluminium alloys. A unique material model for hot deformation of aluminium alloys is proposed in this paper, based on consideration of two processes: (i) increase of dislocation density, induced by plastic deformation, leading to generation, rotation and migration of low angle grain boundaries (LABs) and their transformation into high angle grain boundaries (HABs); (ii) migration of HABs leading to annihilation of both LABs and HABs. At large strain, the above counteracting processes, guided by different mechanisms, lead to saturation of HABs fraction. The model is applied to hot deformation of AA5052 and AA7050 alloys under various temperatures and strain rates, and calculated flow stress, HABs fraction and grain size evolution for both alloys agree well with the corresponding experimental data. The capability of predicting saturation of HABs fraction and average subgrain misorientation angle of both alloys under large strains demonstrate the potential applicability of the model to a wide range of hot forming process conditions.
\end{abstract}

Keywords: Subgrain rotation; Grain boundary migration; Fraction of high angle grain boundary; Continuous dynamic recrystallisation (CDRX); Hot deformation; Aluminium alloys 


\section{Nomenclature}

\begin{tabular}{|c|c|}
\hline Symbol & Definition \\
\hline$A_{i}$ & Material constants $(i=1 \sim 2)$ \\
\hline $\boldsymbol{B}$ & Work hardening coefficient \\
\hline $\boldsymbol{b}$ & Burgers vector \\
\hline$C_{\alpha_{i}}$ & Pre-exponential coefficients of $\alpha_{i}(i=1 \sim 5)$ \\
\hline$c_{\boldsymbol{i}}$ & Material constants $(i=0 \sim 5)$ \\
\hline $\boldsymbol{D}$ & distance between two dislocations \\
\hline$D_{b 0}$ & Grain boundary self-diffusion coefficient \\
\hline$d, d_{0}$ & Grain size and its initial value, $d=2 / S_{H A B}$ \\
\hline $\boldsymbol{E}$ & Elastic modulus \\
\hline$E_{a}$ & Stored energy of subgrain boundary migration \\
\hline$E_{0}$ & Misorientation independent free energy associated with subgrain rotation \\
\hline $\boldsymbol{E}_{\boldsymbol{c}}$ & Stored energy of grain boundary migration with critical misorientation \\
\hline$f_{H A B}$ & Fraction of HABs \\
\hline$G, G_{0}$ & Elastic shear modulus and its initial value \\
\hline $\boldsymbol{h}$ & Strain hardening parameter \\
\hline $\boldsymbol{H}$ & Internal variable representing isotropic hardening of material \\
\hline $\boldsymbol{k}$ & Initial yield stress, temperature dependent \\
\hline $\boldsymbol{k}_{B}$ & Boltzmann constant \\
\hline$K_{g}$ & Constant for calculating shear modulus \\
\hline$M, M^{\prime}$ & Mobility of HABs and LABs respectively \\
\hline$n$ & Material constant, $n=\theta_{c} / \theta_{m}$ \\
\hline$n_{0}$ & Number of dislocation sets \\
\hline $\boldsymbol{P}, \boldsymbol{P}^{\prime}$ & Driving force for migration of $\mathrm{HABs}$ and LABs respectively \\
\hline$Q_{a}$ & Thermal activation energy \\
\hline$Q_{b}$ & Activation energy for grain boundary diffusion \\
\hline$r$ & Dynamic recovery parameter \\
\hline $\boldsymbol{R}$ & Gas constant \\
\hline$S$ & Total area of crystallite boundaries per unit volume \\
\hline$S_{H A B}, S_{H A B 0}$ & Area of high angle grain boundaries per unit volume and its initial value \\
\hline$S_{l a b}$ & Area of low angle grain boundaries per unit volume \\
\hline$T$ & Temperature \\
\hline $\boldsymbol{V}$ & Swept volume by mobile boundaries \\
\hline$v, v^{\prime}$ & Migration velocity of HABs and LABs respectively \\
\hline$V_{m}$ & The molar volume \\
\hline$\alpha$ & Fraction of removed dislocations that forms new LABs \\
\hline$\alpha_{i}$ & Temperature dependent coefficients $(i=1 \sim 5)$ \\
\hline$\beta_{i}$ & Coefficients, temporary use only $(i=1 \sim 5)$ \\
\hline$\gamma_{i}$ & Exponential coefficients $\left(i=1,2, s, \alpha, \theta, l a b, H A B, \alpha_{3}, \alpha_{4}, \alpha_{5}\right)$ \\
\hline
\end{tabular}




$\begin{array}{ll}\gamma_{l a \boldsymbol{b}}^{\prime} & \text { Exponential coefficient, temporary use only } \\ \boldsymbol{\delta} & \text { Subgrain size, } \delta=2 / S_{\text {lab }} \\ \boldsymbol{\varepsilon}, \boldsymbol{\varepsilon}_{\boldsymbol{p}}, \boldsymbol{\varepsilon}_{\boldsymbol{T}} & \text { Strain, plastic strain and total strain respectively } \\ \boldsymbol{\theta}_{\mathbf{0}} & \text { Lower bound misorientation angle for LABs } \\ \boldsymbol{\theta}_{\boldsymbol{c}} & \text { Critical misorientation angle for HABs, } \theta_{c}=15^{0} \text { In this paper } \\ \boldsymbol{\theta}_{\boldsymbol{m}} & \text { Grain boundary with maximum stored energy } \\ \boldsymbol{\theta}_{\boldsymbol{r}} & \text { Comparative misorientation angle, } \theta_{r}=\theta_{c} / \theta_{\text {sat }} \\ \boldsymbol{\theta}_{\boldsymbol{s a t}} & \text { Saturated subgrain misorientation, } \theta_{\text {sat }}=4^{0} \sim 8^{0} \text { for aluminium alloys } \\ \boldsymbol{\theta}_{\boldsymbol{s u b}}, \overline{\boldsymbol{\theta}}_{\boldsymbol{s u b}} & \text { Subgrain misorientation angle and its normalised form, } \bar{\theta}_{\text {sub }}=\theta_{\text {sub }} / \theta_{c} \\ \boldsymbol{v} & \text { Poisson's ratio } \\ \boldsymbol{\rho}, \boldsymbol{\rho}_{\boldsymbol{i}}, \boldsymbol{\rho}_{\boldsymbol{s}} & \text { Average dislocation density, its initial value and saturated value } \\ \overline{\boldsymbol{\rho}} & \text { Normalised dislocation density } \\ \boldsymbol{\rho}_{\boldsymbol{L A B}} & \text { Average dislocation density within LABs } \\ \boldsymbol{\sigma} & \text { Stress } \\ \boldsymbol{\Psi} & \text { Constant that depends on grain shape } \\ \boldsymbol{\Omega}_{\boldsymbol{a}} & \text { G-atom volume } \\ \boldsymbol{\omega} & \text { Dislocation climb mobility } \\ \boldsymbol{d} \boldsymbol{X}, \dot{\boldsymbol{X}}, \overline{\boldsymbol{X}} & \text { Increment, time derivative and normalised value of any variable } X \text { respectively }\end{array}$

\section{Introduction}

Constitutive models for microstructure-property relations of aluminium alloys have been proposed and developed for various scales and different processing aspects. Research at molecular or nano scale can account for the shape and size distribution of precipitates and nano particles through molecular dynamics models, for example, for plastic forming (Krasnikov et al., 2020) and compaction (Mayer et al., 2020). Precipitates and particles play an important role in change in dislocation density during forming and resultant material properties. Larger scale models, such as crystal plasticity (CP) and cellular automata (CA) models, are widely used to predict microstructure and corresponding mechanical properties of alloys, arising from mechanical processing. Grain boundary dislocation, point defect, grain boundary slide and climb can all be taken into consideration in these models, and changes in material properties, such as hardening, softening and rate sensitivity behaviour can be well predicted (Hu et al., 2016; Khan and Liu, 2012; Khan et al., 2015; Liu et al., 2019). Currently, increasing attention is being paid to the coupling method to extend model application to multiscale fields, such as determination of mechanical response and subsequent plastic deformation with consideration of grain level dynamic recrystallisation (DRX), by using the coupled $\mathrm{CP} / \mathrm{CA}$ approach ( $\mathrm{Li}$ et al., 2016; Nagra et al., 2020).

The models referred to above mainly focus on small scale and a microstructure-based statistically equivalent representative volume element (RVE) model is often required (Majta et 
al., 2016; Tu et al., 2019). It is difficult to apply such models to simulations of complete hot deformation processes and to do this an accurate phenomenological or physically based micro/macro scale material model is required. To date, several micro/macro scale models have been developed; such as the phenomenological hardening model (Nayan et al., 2019), physically based multi-step stress relaxation model (Zheng et al., 2018) and creep age forming model (Li et al, 2017), physically based mean field model (Neumann and Böhlke, 2016), physically based visco-plastic self-consistent (VPSC) model (Tang et al., 2019) and dislocation density based constitutive model (Lin et al., 2018). Although these models have a significant role in forming process simulation, it is thought that, using them, interpretation of extensive changes of microstructure, arising in work-pieces during hot, dynamic deformation processes, is difficult (Lin and Chen, 2011) and a physically based DRX model would be highly beneficial. The work described in this paper is concerned with a DRX model for aluminium alloy.

In general, during hot deformation, DRX can occur either as: discontinuous dynamic recrystallisation (DDRX) (Castro-Fernandez and Sellars, 1988; Shi et al., 2014), or geometric dynamic recrystallisation (GDRX) (Kassner and McMahon, 1987; Pettersen et al., 2003), or continuous dynamic recrystallisation (CDRX) (Gourdet and Montheillet, 2003; Sun et al., 2018). DDRX may occur in high purity aluminium and in aluminium alloys that contains large particles $(>1 \mu \mathrm{m})$ (Gourdet and Montheillet, 2000), but is prevented by the presence of high densities of fine particles and precipitates (Rokni et al., 2011). McQueen and co-workers maintained that there was no evidence of DDRX occurrence during the large-strain hot deformation of a wide range of aluminium and its alloys such as pure aluminium (Kassner and McMahon, 1987) and Al-5.8at\%Mg (Henshall et al., 1992) and that GDRX, instead, dominated the DRX process. Under large strain ( $\varepsilon$, often above 3), GDRX arose and developed in the form of serrated high angle grain boundaries (HABs), leading to a steady-state fraction of HABs $\left(f_{H A B}\right)$.

More commonly, CDRX has been observed in many hot forming processes of aluminium alloys, such as equal-channel angular extrusion of Al-Li-Mg-Sc alloy (Kaibyshev et al., 2005), hot torsion of Al-Mg-Si alloy (even at a strain of about 20) (Lee et al., 2016), and friction stir welding of AA6082-T6 alloys (Fratini and Buffa, 2005). Driver (2018) confirmed the existence of CDRX and suggested that a temperature window of $0.5 T_{m}<T<0.7 T_{m}$ favours CDRX for most aluminium alloys. Recently, Sun et al. (2018) studied hot deformation of as-extruded AA7075 alloys at a strain of 0.8 and deformation temperature above $0.6 T_{m}$, and observed four characteristics within the recrystallised microstructure: $(i)$ recrystallised grain size is similar to that of the subgrains; ( $i i$ ) orientations of recrystallised grains are different from those of original grains; (iii) recrystallised grains are concentrated along the original grain boundaries; (iv) a large number of LABs exist after recrystallisation.

CDRX is much more complicated than DDRX and GDRX (Sun et al., 2018) and few CDRX models have been developed. The most well-known model for CDRX is the GM model, first proposed by Gourdet and Montheillet (2003), in which the whole recrystallisation process is 
described as: ( $i$ ) new LABs are formed due to the condensation and rearrangement of dislocations during recovery, (ii) part of LABs is transformed into HABs, as absorption of dislocations in pre-existing boundaries leads to an increase of misorientation, (iii) dislocation density can be annihilated by migration of HABs. Flow stress and associated evolution of microstructure, including subgrain size and dislocation density, can be predicted by the GM model.

However, this GM model has not been validated by quantitative comparison with experimental results. Criticism of the model by McQueen and Kassner (2004) include: (i) no mechanism to eliminate dislocations from LABs, because their migration and rearrangement was neglected. Both phenomena were clearly observed and seen to vary with hot deformation conditions; ( $i$ i) neither saturation of LABs misorientation angle $\left(4^{\circ} \sim 8^{\circ}\right)$, reported by Furu and Nes (1996), nor saturation of the fraction of high angle grain boundaries $\left(f_{H A B}\right)$ can be predicted by the model.

More recently, Maizza et al. (2018) extended the GM model and applied it to aluminium alloy 5052, where $f_{H A B}$ was not theoretically quantified, but instead determined by exponential fitting through the $f_{H A B}-\varepsilon$ curve. Flow stress curves fitted well with the experimental results, but microstructure evolution mechanisms, e.g., change in HABs area which directly yield grain size, is missing. Sun et al. (2018) modified the GM model by including dislocation density, subgrain boundary area, recrystallised grain boundary area, high angle grain boundary area and subgrain boundary misorientation angle as internal-state variables. A set of constitutive models for AA7075 alloys was established to calculate flow stress, grain size and average misorientation angle for the softening stage. The model predicted the trend of flow stress in the softening stage and the substructures fairly well, but no comparison was provided for the hardening stage which is an important aspect of the hot deformation process.

The purpose of the research described in this paper is development of a new set of CDRXbased equations to describe viscoplastic flow behaviour and microstructural evolution of aluminium alloy hot formed within a wide strain range. The model considers dislocation accumulation due to deformation, formation and migration of LABs, rotation of subgrains, and migration of HABs as well transformation of LABs to HABs.

\section{Mechanisms of microstructural evolution}

Fig. 1 illustrates the mechanisms that contribute to microstructural evolution and viscoplastic flow behaviour of aluminium alloy during hot forming, that are considered in the current study: 

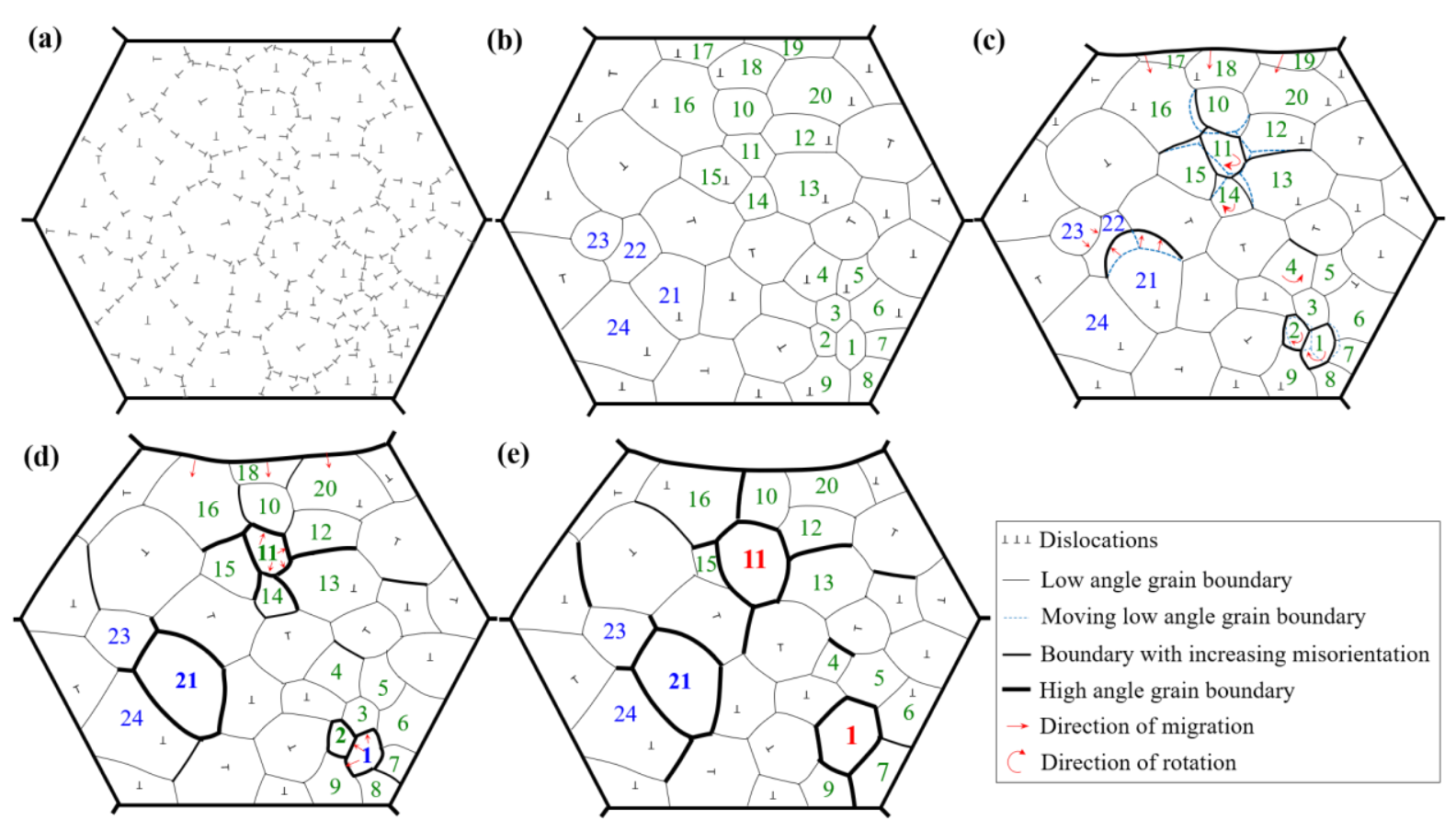

Fig. 1 Schematic illustration of microstructural evolution during deformation: (a) dislocation accumulation; (b) formation of LABs and subgrains; (c) increase of LABs misorientation due to subgrain rotation and LABs migration, and reduction of LABs due to HABs and LABs migration; (d) formation of HABs from LABs; (e) reduction of LABs and HABs due to HABs migration.

(1) Accumulation of dislocations during the forming process (Fig. 1(a)) results in the formation of new subgrains with low angle grain boundaries (Fig. 1(b)) (Gourdet and Montheillet, 2003);

(2) Migration and agglomeration of like-sign dislocations in the LABs, followed by grain boundary shearing, leads to the rotation of subgrains, e.g., subgrains 1, 2, 4, 11 and 14 in Fig. 1(c) (Fazan et al., 1954);

(3) Rotation of subgrains leads to an increase of the misorientation angle and eventually transforms part of LABs to HABs, e.g., (sub)grain 11 in Figs. 1(b), 1(c) and 1(d) (Li, 1962; Gourdet and Montheillet, 2003; Mishra et al., 2007);

(4) Migration of LABs absorbs dislocations from other LABs and leads to an increase of misorientation, gradually turning part of LABs into HABs, e.g., LABs of subgrain 21 in Figs. 1(b) and 1(c) to HABs of grain 21 in Fig. 1(d) (Blum et al., 1995; Rios et al., 2005);

(5) Migration of HABs sweeps part of HABs and LABs, leading to a decrease of the corresponding areas. For example, migration of the original grain boundary (HABs) results in the annihilation of LABs surrounding subgrains 17 and 19 from Fig. 1(c) to Fig. 1(d); migration of the newly formed HABs leads to the annihilation of LABs that surround subgrain 3 from Fig. 1(d) to Fig. 1(e); Migration of the HABs sweeps part of the HABs and LABs that surround grain 11 from Fig. 1(d) to Fig. 1(e).

Here LABs are classically defined by misorientation angle of between $2^{\circ}$ and $15^{\circ}$, while 
HABs are defined by a misorientation of no less than $15^{\circ}$ (Sun et al., 2018; Zhao et al., 2018; Beucia et al., 2019). Subgrains are those surrounded by LABs; when misorientation angles of all surrounding LABs increase to $15^{\circ}$, the subgrains are regarded as independent grains (Poirier and Nicolas, 1975). The area of HABs $\left(S_{H A B}\right)$ and area of LABs $\left(S_{l a b}\right)$ in this paper are respectively symbols for average area of $\mathrm{HABs}$ per unit volume and average area of LABs per unit volume. In this study, it is assumed that: (i) mobility of LABs is misorientation and temperature dependent, while mobility of HABs is dependent on temperature but independent of misorientation; (ii) average grain size $(d)$ and average subgrain size $(\delta)$ can be calculated using a stereological relationship from area of HABs $\left(S_{H A B}\right)$ and area of LABs $\left(S_{l a b}\right)$ respectively, i.e., $d=2 / S_{H A B}$ and $\delta=2 / S_{\text {lab }}$ (Gourdet and Montheillet, 2003); (iii) total area of boundaries (S) is the sum of area of HABs and area of LABs $\left(S_{l a b}\right): S=S_{H A B}+S_{l a b}$.

From above, it can be understood that the mechanisms considered in the proposed model include dislocation accumulation, formation and migration of LABs, subgrain rotation, HABs migration and transformation of LABs to HABs. All these processes are interrelated and can affect one another.

\section{CDRX-based material model for aluminium alloy}

\subsection{Dislocation density evolution model}

Static recovery and static recrystallisation can occur in the absence of plastic deformation. During hot deformation, dynamic recovery (DRV) and dynamic recrystallisation (DRX) are the main softening mechanisms. Together with strain hardening, they control evolution of mechanical properties and microstructure, including dislocation density which is the subject of modelling in this section.

Because of the constraints imposed by TEM and the more recent synchrotron X-ray Laue microdiffraction facilities (Verheyden et al., 2019), it is very difficult to accurately measure evolution of dislocation density $(\rho)$ during hot deformation and to obviate the problem, Lin and Liu (2003) proposed a dislocation evolution model, by using a normalised dislocation density $\bar{\rho}:$

$$
\dot{\bar{\rho}}^{+}=c_{0}\left(S_{H A B 0} / S_{H A B}\right)^{\gamma_{s}}(1-\bar{\rho}) \dot{\varepsilon}_{p}-\alpha_{1} \bar{\rho}^{c_{1}}
$$

where the "+" sign indicates that it is positively contributing to the total dislocation density. $S_{H A B O}$ is the initial aera of HABs $\left(S_{H A B O}=2 / d_{0}\right.$ in which $d_{0}$ is the initial average grain size). $\bar{\rho}$ is the normalised dislocation density and is defined as $\left(\rho-\rho_{i}\right) / \rho_{s}$ where $\rho_{i}$ is dislocation density before deformation (often annealed state) and $\rho_{s}$ is the saturated dislocation density arising during hot deformation. Some experimentally measured values have been reported previously for both $\rho_{i}$ and $\rho_{s}$. The initial dislocation density in annealed aluminium alloys is in the order of $10^{10} \mathrm{~m}^{-2}$ (Beucia et al., 2019; Chen et al., 2020). During deformation, dislocation density increases rapidly with strain by 2 or 3 orders of magnitude 
and finally reaches a steady-state value of above $10^{13} \mathrm{~m}^{-2}$ (Baxter et al., 1999; Beucia et al., 2019). At the beginning of deformation, $\rho=\rho_{i}$, and the initial normalised dislocation density $\bar{\rho}=0$. As plastic deformation progresses, dislocation density increases and eventually saturates when $\rho_{s} \gg \rho_{i}$. Thus the normalised dislocation density will increase proportionally with $\rho$ as strain increases and at saturation, is approximately equal to 1 . Because the value of initial density is relatively very low it can be neglected with little loss to accuracy. Therefore, normalised dislocation density $\bar{\rho}$ varies from 0 to 1 and by using it, the exact $\rho$ value is not required for the proposed model, eliminating the need for difficult measurement. Nevertheless, measurements of dislocation density may be necessary for other purposes and previous studies have shown that $\rho_{s} \gg \rho_{i}$.

In the first term on the right-hand side (rhs) of Eq. (1), $(1-\bar{\rho}) \dot{\varepsilon}_{p}$, represents the development of dislocation density due to plastic strain and dynamic recovery (Li et al., 2009). Dislocation density initially increases with plastic strain and later the increasing rate decreases due to dynamic recovery (Lin, et al., 2005). $\left(S_{H A B 0} / S_{H A B}\right)^{\gamma_{S}}$ represents the effect of grain size on dislocation evolution, i.e. for a given strain, fewer dislocations are generated the smaller is the grain size.

Through analysis of the temperature and strain rate dependence of strain hardening, for $\mathrm{Al}$, $\mathrm{Ti}$, and $\mathrm{Cu}$ alloys, Kreyca and Kozeschnik (2018) found that the dislocation evolution depends on temperature and strain rate during dynamic recovery and thermal activation contributes to dislocation annihilation. This is written as the second term on the rhs of Eq. (1) as, $\alpha_{1} \bar{\rho}^{c_{1}}$. Here $\alpha_{1}=c_{\alpha 1} \exp \left(-\frac{Q_{a}}{R T}\right)$ represents thermal activation, where $c_{\alpha 1}$ is a material constant, $Q_{a}, R$, and $T$ are respectively, thermal activation energy, gas constant and absolute temperature.

Dynamic recrystallisation decreases dislocation density within the material and contributes to the change of dislocation density in the model. Gourdet and Montheillet (2003) proposed a model to calculate dislocation annihilation during dynamic recrystallisation, which was described as grain boundary migration. In the model, annihilated dislocations, represented as $d \rho^{-}$, (the '-' sign shows it negatively contributes to dislocation density) during a strain increment $(d \varepsilon)$ was given as:

$$
d \rho^{-}=\rho d V
$$

From Eq. (2), dislocations located in the volume $d V$ swept by the mobile boundaries will be annihilated. During a time-increment $d t, d V$ is the product of the total area per unit volume $(S)$ with the swept distance $(v d t), d V=S \cdot v d t$, where $v$ is the grain boundary migration speed. Therefore, the reduction of dislocation density is:

$$
\dot{\rho}^{-}=\rho \cdot S v
$$

The grain boundary migration speed $v$ is the product of the migration driving force $P$ and the mobility of the grain boundary $M$, i.e:

$$
v=M P
$$

where the grain boundary mobility: 


$$
M=\frac{V_{m} D_{b 0}}{b k_{B} T} \exp \left(-\frac{Q_{b}}{R T}\right)
$$

and the driving force $P$ for grain boundary movement per unit area:

$$
P=\rho G b^{2}
$$

in which $b, D_{b 0}, Q_{b}, k_{B}, V_{m}$ are respectively the Burgers vector, the pre-exponential factor of the grain boundary self-diffusion coefficient, the activation energy of grain boundary diffusion, the Boltzmann constant and the molar volume. $G$ is the shear modulus, $G=$ $G_{0} \exp \left(-K_{g} \cdot T\right), G_{0}=2.99 \times 10^{10} \mathrm{~Pa}, K_{g}=5.4 \times 10^{-4} \mathrm{~K}^{-1}$ for aluminium alloys (Duan and Sheppard, 2002).

Then, the grain boundary migration speed $v$ can be given as:

$$
v=M P=\frac{V_{m} D_{b 0}}{b k_{B} T} \exp \left(-\frac{Q_{b}}{R T}\right) \cdot \rho b^{2} G_{0} \exp \left(-K_{g} \cdot T\right)=\beta_{1} \rho
$$

where $\beta_{1}=\frac{b V_{m} G_{0} D_{b 0}}{k_{B} T} \exp \left(-K_{g} \cdot T-\frac{Q_{b}}{R T}\right)$. From above, Eq. (3) can now be written as:

$$
\dot{\rho}^{-}=\beta_{1} S \rho^{2}
$$

Given the definition of the normalised dislocation density, $\bar{\rho}=\left(\rho-\rho_{i}\right) / \rho_{s}$, considering $\rho_{s} \gg \rho_{i}, \rho$ can be approximated as proportional to $\bar{\rho}$. Although this does introduce an error, as stated above, $\rho_{i} / \rho_{s} \approx 10^{-3}$, thus the error is small. To be more general, a term $\beta_{3} \bar{\rho}^{\gamma_{\alpha}}$ is used to replace the term $\rho^{2}$, where $\gamma_{\alpha}$ is an adjustable constant for different alloys. Thus Eq. (8) becomes:

$$
\dot{\bar{\rho}}^{-}=\alpha_{2} S \bar{\rho}^{\gamma_{\alpha}}
$$

where $\alpha_{2}=C_{\alpha_{2}} \frac{1}{T} \exp \left(-K_{g} \cdot T-\frac{Q_{b}}{R T}\right)$ is a material constant in which $C_{\alpha_{2}}=\frac{\beta_{3}}{\beta_{2}} \frac{b V_{m} G_{0} D_{b 0}}{2 k_{B} T}$.

Therefore, combining Eq. (1) and Eq. (9) to consider the dislocation accumulation, dynamic recovery and dynamic recrystallisation, the dislocation density evolution model can be written as:

$$
\dot{\bar{\rho}}=\dot{\bar{\rho}}^{+}-\dot{\bar{\rho}}^{-}=c_{0}\left(S_{H A B 0} / S_{H A B}\right)^{\gamma_{S}}(1-\bar{\rho}) \dot{\varepsilon}_{p}-\alpha_{1} \bar{\rho}^{c_{1}}-\alpha_{2} S \bar{\rho}^{\gamma_{\alpha}}
$$

\subsection{Low angle grain boundary area evolution model}

New low angle grain boundaries are formed through dislocation accumulation and recombination during the plastic deformation $\left(\dot{S}_{\text {lab }}^{+}\right)$. Contrariwise, subgrain rotation leads to a reduction of LABs $\left(\dot{S}_{l a b}^{-(1)}\right)$. In addition, subgrain boundary migration, which is thermally assisted and has been observed in pure aluminium and Al-5\% Mg alloy (Blum et al., 1995), induces growth of newly formed subgrains at the expense of its neighbours $\left(\dot{S}_{l a b}^{-(2)}\right)$. Moving low angle grain boundaries absorb dislocations, increasing their orientation differences, and their energy and mobility until they are transformed into HABs (Rios et al., 2005). Meanwhile, 
migration of HABs may sweep and annihilate part of LABs $\left(\dot{S}_{l a b}^{-(3)}\right)$. Therefore, change in area of LABs can be expressed as the sum of the increase due to new LABs and reduction due to rotation and migration:

$$
\dot{S}_{l a b}=\dot{S}_{l a b}^{+}-\dot{S}_{l a b}^{-(1)}-\dot{S}_{l a b}^{-(2)}-\dot{S}_{l a b}^{-(3)}
$$

As described by Gourdet and Montheillet (2003), for a given LAB of misorientation $\theta_{\text {sub }}$, the classical relationship $\theta_{\text {sub }}=b / D$, where $D$ is the distance between two dislocations, results in a dislocation length per unit area $L_{\theta}=n_{0} \theta_{\text {sub }} / b$, where $n_{0}$ is the number of dislocation sets in the boundary. Thus, dislocation length per unit volume, or dislocation density is given by $\rho=S_{l a b} n_{0} \theta_{\text {sub }} / b$, or $d \rho=d S_{l a b} n_{0} \theta_{\text {sub }} / b$. This function illustrates that, to result in an increase of $d S_{l a b}$ with the average misorientation of $\theta_{\text {sub }}$, an incremental increase of $d \rho$ must be absorbed.

The dislocation density, reduced by dynamic recovery, is given as, $d \rho_{r e c}=r \rho d \varepsilon_{p}$ in the Laasraoui-Jonas equation (Laasraoui and Jonas, 1991a), where $r$ is the dynamic recovery parameter. Then, a fraction $(\alpha)$ of the removed dislocation density $\left(\alpha d \rho_{\text {rec }}=\alpha r \rho d \varepsilon_{p}\right)$ is consumed to form the new LABs $\left(d S_{\text {lab }}^{+}\right)$with an average misorientation angle of $\theta_{0}$ :

$$
\alpha r \rho d \varepsilon_{p}=d S_{l a b}^{+} n_{0} \theta_{0} / b
$$

which can be rewritten in a rate form:

$$
\dot{S}_{l a b}^{+}=\left(\frac{b}{n_{0} \theta_{0}}\right) \alpha r \rho \dot{\varepsilon}_{p}
$$

Due to subgrain rotation, misorientation angle of LABs increases (with a rate of $\dot{\theta}_{\text {sub }}$ ). Part of LABs obtains sufficient increase of misorientation to change to HABs and thus reduces the area of LABs. This process can be modelled as (Sun et al., 2018):

$$
\dot{S}_{l a b}^{-(1)}=c_{02} S_{l a b} \dot{\theta}_{\text {sub }}
$$

where $c_{02}$ is a coefficient associated with the part of LABs that is turned to HABs.

The second mechanism of area reduction of LABs is their migration. During a timeincrement $d t$, the volume swept by LABs $d V_{\text {lab }}$ is the product of the area per unit volume $S_{l a b}$ with the swept distance $v^{\prime} d t, d V_{l a b}=S_{l a b} \cdot v^{\prime} d t$, where $v^{\prime}$ is the LABs migration velocity. Therefore, this aspect of the reduction in LABs area is given by:

$$
\dot{S}_{l a b}^{-(2)}=S_{l a b} \cdot \dot{V}_{l a b}=S_{l a b} \cdot S_{l a b} v^{\prime}
$$

where $v^{\prime}=M^{\prime} P^{\prime}$, in which $M^{\prime}$ and $P^{\prime}$ are respectively the low angle grain boundary migration mobility and the driving force per unit area on the boundaries.

As emphasised by Humphreys and Hatherly (2012), the mobility of LABs increases rapidly with the misorientation until the critical misorientation value $\theta_{c}\left(\theta_{c}=15^{0}\right)$ is reached, then it becomes misorientation independent. Thus, the mobility of the boundaries can be written as a function of misorientation angle: 


$$
M^{\prime}=\beta_{4}\left(\frac{\theta_{\text {sub }}}{\theta_{c}}\right)^{\gamma_{l a b}^{\prime}} M
$$

where $\gamma_{l a b}^{\prime}$ and $\beta_{4}$ are constants. A similar relationship between $M^{\prime}$ and $M$ with $\gamma_{l a b}^{\prime} \approx$ 5.18 was reported by Huang and Humphreys (2000).

Ignoring precipitation and second-phase particles, the main driving force for recrystallisation due to LABs migration is the stored energy during straining in the form of crystalline defects (Rios et al., 2005), and can be expressed as (Dillamore et al., 1967):

$$
P^{\prime}=\frac{\Psi \Omega_{a}}{\delta} E_{a}
$$

where $\Psi$ is a numerical constant depending on grain shape; $\Omega_{a}$ is the gram-atomic volume; $\delta$ is the average subgrain size; and $E_{a}$ is the stored energy of the subgrain boundary, which can be calculated through the Read-Shockley formula (Read and Shockley, 1950). A comparative form of the Read-Shockley formula is often convenient for use, where the boundary energy $\left(E_{\alpha}\right)$ and misorientation $\left(\theta_{\text {sub }}\right)$ are normalised with respect to the values of these parameters $\left(E_{c}\right.$ and $\left.\theta_{c}\right)$ when the boundary becomes a high angle boundary (i.e. $\theta_{c}=$ 15º (Dillamore et al., 1967; Humphreys and Hatherly, 2012):

$$
E_{a}=E_{c} \frac{\theta_{\text {sub }}}{\theta_{c}}\left(1-\ln \left(\frac{\theta_{\text {sub }}}{\theta_{c}}\right)\right)
$$

Then Eq. (17) becomes:

$$
P^{\prime}=\left[\left(\frac{\theta_{s u b}}{\theta_{c}}\right)\left(1-\ln \left(\frac{\theta_{s u b}}{\theta_{c}}\right)\right)\right] P
$$

Substituting Eq. (16) and Eq. (19) into Eq. (15), the area reduction rate of LABs due to LABs migration can be expressed as:

$$
\dot{S}_{l a b}^{-(2)}=\beta_{4} S_{l a b}^{2}\left(\frac{\theta_{\text {sub }}}{\theta_{c}}\right)^{\gamma_{\theta}}\left(1-\ln \left(\frac{\theta_{\text {sub }}}{\theta_{c}}\right)\right) M P
$$

where $\gamma_{\theta}$ is a material constant, $\gamma_{\theta}=\gamma_{l a b}^{\prime}+1 \approx 6.18$.

The third mechanism by which LABs area is reduced is migration of HABs. During a timeincrement $d t$, the volume swept by HABs $d V_{H A B}$ is the product of the area per unit volume $\left(S_{H A B}\right)$ with the swept distance $(v d t), d V_{H A B}=S_{H A B} v d t$, where $v$ is the grain boundary migration speed. For HABs, $v$ is independent of misorientation angle (Humphreys and Hatherly, 2012). Therefore, the reduction of $S_{l a b}$ from the swept volume by HABs can be given as:

$$
\dot{S}_{l a b}^{-(3)}=S_{l a b} \cdot \dot{V}_{H A B}=S_{l a b} \cdot S_{H A B} v=S_{l a b} S_{H A B} \cdot M P
$$

Therefore, the evolution of LABs area becomes

$$
\dot{S}_{l a b}=\left(\frac{b}{n_{0} \theta_{0}}\right) \alpha r \rho \dot{\varepsilon}_{p}-c_{02} S_{l a b} \dot{\theta}_{s u b}-\alpha_{3} \rho S_{l a b}^{2}\left(\frac{\theta_{s u b}}{\theta_{c}}\right)^{\gamma \theta}\left(1-\ln \left(\frac{\theta_{\text {sub }}}{\theta_{c}}\right)\right)-\alpha_{5} \rho S_{l a b} S_{H A B}
$$

Using the normalised dislocation density and for convenience, introducing a normalised misorientation angle, $\bar{\theta}_{\text {sub }}=\theta_{\text {sub }} / \theta_{c}$, the evolution law of the LABs area can be written in a simpler form:

$$
\dot{S}_{l a b}=\alpha_{4} \bar{\rho}^{\gamma_{1}} \dot{\varepsilon}_{p}-c_{2} S_{l a b} \dot{\bar{\theta}}_{s u b}-\alpha_{3} S_{l a b}^{2} \bar{\theta}_{\text {sub }}^{\gamma_{\theta}}\left(1-\ln \left(\bar{\theta}_{\text {sub }}\right)\right) \bar{\rho}^{\gamma_{l a b}}-\alpha_{5} S_{l a b} S_{H A B} \bar{\rho}^{\gamma_{H A B}}
$$


where $\quad c_{2}=\theta_{c} c_{02} \quad, \quad \alpha_{3}=\frac{C_{\alpha_{3}}}{T} \exp \left(-K_{g} \cdot T-\frac{Q_{b}}{R T}\right), \quad \alpha_{4}=C_{\alpha_{4}} \exp \left(-\frac{Q_{a}}{R T}\right) \quad, \quad \alpha_{5}=$ $C_{\alpha_{5}} \exp \left(-\frac{Q_{a}}{R T}\right) . C_{\alpha_{3}}, C_{\alpha_{4}}, C_{\alpha_{5}}, c_{2}, \gamma_{1}, \gamma_{\theta}, \gamma_{l a b}, \gamma_{H A B}$ are material constants. Temperature dependent coefficient $\alpha_{4}$ indicates that the dynamic recovery process is thermally activated. As discussed in section 2, boundaries with misorientation angle larger than $\theta_{c}\left(\theta_{c}=15^{0}\right)$ are regarded as HABs, and boundaries with misorientation angle between $2^{0} \sim 15^{0}$ are treated as LABs, therefore $\bar{\theta}_{\text {sub }}$ value is in the range of $2 / 15 \sim 1.0$.

\subsection{High angle boundary area evolution model}

Both rotation of subgrains and migration of LABs transform part of LABs into HABs. The second and third terms on the rhs of Eq. (23) illustrate the formation of HABs due to subgrain rotation and LABs migration respectively, and these HABs form one part of the change in HABs area per unit volume:

$$
\dot{S}_{H A B}^{+}=c_{2} S_{l a b} \dot{\bar{\theta}}_{\text {sub }}+\alpha_{3} S_{\text {lab }}^{2} \bar{\theta}_{\text {sub }}^{\gamma_{\theta}}\left(1-\ln \left(\bar{\theta}_{\text {sub }}\right)\right) \bar{\rho}^{\gamma_{l a b}}
$$

Migration of HABs sweeps part of pre-existing and newly formed HABs and annihilates those swept ones. During a time-increment $d t$, the swept volume $d V_{H A B}=S_{H A B} v d t$. Therefore, the reduction of $S_{H A B}$ due to migration of HABs can be given as:

$$
\dot{S}_{H A B}^{-}=S_{H A B} \dot{V}_{H A B}=S_{H A B} \cdot S_{H A B} \cdot v=S_{H A B}^{2} \cdot M P
$$

which can be transformed using a normalised dislocation form, with the same way as for Eq. (7) to Eq. (9),

$$
\dot{S}_{H A B}^{-}=\alpha_{2} S_{H A B}^{2} \bar{\rho}^{\gamma_{H A B}}
$$

then, the high angle boundary area evolution model can be expressed as:

$$
\dot{S}_{H A B}=c_{2} S_{l a b} \dot{\bar{\theta}}_{\text {sub }}+\alpha_{3} S_{\text {lab }}^{2} \bar{\theta}_{\text {sub }}^{\gamma_{\theta}}\left(1-\ln \bar{\theta}_{\text {sub }}\right) \bar{\rho}^{\gamma_{l a b}}-\alpha_{2} S_{H A B}^{2} \bar{\rho}^{\gamma_{H A B}}
$$

The first two terms on the rhs of Eq. (27) represent HABs transformed from LABs by the subgrain rotation and the LABs migration, and the last term is the annihilation of HABs during the process of HABs migration.

\subsection{Subgrain rotation model}

The classic relationship between misorientation angle $\theta_{\text {sub }}$, dislocation spacing $D$ and the Burgers vector $b$ is $\theta_{\text {sub }}=b / D$, where dislocation spacing $D=\rho^{-1 / 2}$. Thus $\theta_{\text {sub }}=b \rho^{1 / 2}$, where $d \rho$ represents dislocations assimilated by subgrain boundaries, and can be defined as, $d \rho=\beta_{5} \rho d \varepsilon_{p}$, where $\beta_{5}=\dot{\varepsilon} \exp \left(-Q_{a} / R T\right)$ is a temperature and strain rate dependent parameter (Sun, 2018). Then, the subgrain rotation model can be expressed as:

$$
\dot{\theta}_{\text {sub }}=\frac{1}{2} b \rho^{-1 / 2} \dot{\rho}=\frac{1}{2} \beta_{5} b \rho^{1 / 2} \dot{\varepsilon}_{p}
$$

Eq. (28) indicates that subgrain boundary misorientation increases with absorption of dislocations, and the rotation rate is misorientation independent. However, it has been found that subgrain rotation rate is associated with the subgrain size and boundary misorientation, 
and the Read-Shockley formula (Read and Shockley, 1950) should be introduced (Doherty and Szpunar, 1984; Li, 1962). The derived model for subgrain rotation rate then becomes (Li, 1962):

$$
\dot{\theta}_{\text {sub }}=3 \omega b E_{0} \cdot\left[\theta_{\text {sub }} \ln \left(\theta_{\text {sub }} / \theta_{m}\right) \cdot \delta^{-2}\right]
$$

where the stored energy $E_{0}=G b^{2} / 4 \pi(1-v)$ is independent of misorientation, in which $v$ is Poisson's ratio; $\omega$ is the climb mobility. $\theta_{m}$ is the misorientation angle of grain boundary at which the boundary will have maximum energy. The typical value for $\theta_{m}$ is about $20-25^{\circ}$ ( $\mathrm{Li}$, 1962).

The term, $\theta_{\text {sub }} \ln \left(\theta_{\text {sub }} / \theta_{m}\right) \cdot \delta^{-2}$ in Eq. (29) has been commonly used to illustrate changes of subgrain rotation rate with subgrain size and misorientation angle. Subgrain rotation rate is strongly dependent on the misorientation (Doherty and Szpunar, 1984; Upmanyu et al., 2006). Also, it is inversely proportional to subgrain size as $\dot{\theta}_{s u b} \propto \delta^{-\xi}$, where the exponent coefficient $\xi=2$, as shown by Lobkovsky and Warren (2001) and Upmanyu et al. (2006), who used the phase-field method and the molecular dynamics (MD) simulations. Considering $\delta=$ $2 / S_{\text {lab }}$, the subgrain ration rate is thus proportional to $S_{\text {lab }}^{2}\left(\dot{\theta}_{s u b} \propto S_{\text {lab }}^{2}\right)$.

It should be noted that subgrain rotation rate described in Eq. (29) is always negative as $\theta_{\text {sub }}<\theta_{m}$, which leads to a decreasing subgrain misorientation. In the case of misorientation angle increasing with time as in the proposed model, $\theta_{\text {sub }} / \theta_{m}$ can be replaced by $\theta_{m} / \theta_{\text {sub }}$ (Li, 1962). Thus, Eq. (29) becomes:

$$
\dot{\theta}_{\text {sub }}=3 \omega b E_{0} \cdot\left[\theta_{\text {sub }} \ln \left(\theta_{m} / \theta_{\text {sub }}\right) \cdot \delta^{-2}\right]
$$

Many experiments have found that the average misorientation of subgrains is always saturated at a steady-state value $\left(\theta_{\text {sat }}\right)$ of about $4^{\circ} \sim 8^{\circ}$ for aluminium alloys when they are deformed to a critical strain (Furu and Nes, 1996; McQueen and Kassner, 2004). From this aspect, the normalised subgrain rotation rate will be gradually reduced to zero when it approaches $\theta_{\text {sat }}$. To satisfy this observed phenomenon, an additional factor $\left(1-\theta_{r} \bar{\theta}_{s u b}\right)$ is added, where $\bar{\theta}_{\text {sub }}=\theta_{\text {sub }} / \theta_{c}$ is the normalised misorientation angle and $\theta_{r}=\theta_{c} / \theta_{\text {sat }}$. Combining the above and Eqs. (28) and (30) and using the normalised variables, the increasing rate of $\bar{\theta}_{\text {sub }}$ can be finally written as:

$$
\dot{\bar{\theta}}_{\text {sub }}=\alpha_{6} \dot{\varepsilon}_{p} \bar{\rho}^{\gamma_{2}} S_{\text {lab }}^{2} \bar{\theta}_{\text {sub }}\left(\theta_{r} \bar{\theta}_{\text {sub }}-1\right) \ln \left(n \bar{\theta}_{\text {sub }}\right)
$$

where $n=\theta_{c} / \theta_{m}, \alpha_{6}=C_{6} \exp \left(-\frac{Q_{a}}{R T}\right)$.

\subsection{Viscoplastic flow}

A sinh-law viscoplastic constitutive equation has been used widely for its suitability to a wide range of strain rate and stress levels (Lin and Liu, 2003), and is adopted for this application:

$$
\dot{\varepsilon}_{p}=A_{1} \sinh \left[A_{2}(|\sigma|-H-k)\right] \cdot S_{H A B}^{\gamma_{d}}
$$

where $A_{1}, A_{2}, \gamma_{d}$ are temperature dependent material constants. $\sigma$ and $k$ are flow stress and initial yield stress respectively and $H$ is an internal variable representing isotropic 
hardening. $H$ is directly related to dislocation density and can be described as $H=B \bar{\rho}^{0.5}$ (Mohamed et al., 2012; Xiao et al., 2018). Through differentiation, it becomes:

$$
\dot{H}=\frac{1}{2} B \bar{\rho}^{-0.5} \dot{\bar{\rho}}
$$

Finally, the flow stress $\sigma$ can be calculated by:

$$
\dot{\sigma}=E\left(\dot{\varepsilon}_{T}-\dot{\varepsilon}_{p}\right)
$$

where $\dot{\varepsilon}_{T}$ is the total strain rate and $E$ is the elastic modulus.

\subsection{Constitutive equations}

From the above discourse, the complete set of constitutive equations can be listed as:

$$
\left\{\begin{array}{l}
\dot{\varepsilon}_{p}=A_{1} \sinh \left[A_{2}(|\sigma|-H-k)\right] \cdot S_{H A B}^{\gamma_{d}} \\
\dot{H}=\frac{1}{2} B \bar{\rho}^{-0.5} \dot{\bar{\rho}} \\
\dot{\bar{\rho}}=c_{0}\left(S_{H A B 0} / S_{H A B}\right)^{\gamma_{S}}(1-\bar{\rho}) \dot{\varepsilon}_{p}-\alpha_{1} \bar{\rho}^{c_{1}}-\alpha_{2} S^{\gamma^{\alpha}} \\
\dot{S}_{H A B}=c_{2} S_{l a b} \dot{\bar{\theta}}_{\text {sub }}+\alpha_{3} \bar{\rho}^{\gamma_{l a b}} S_{\text {lab }}^{2} \bar{\theta}_{\text {sub }}^{\gamma_{\theta}}\left(1-\ln \left(\bar{\theta}_{\text {sub }}\right)\right)-\alpha_{2} \bar{\rho}^{\gamma_{H A B}} S_{H A B}^{2} \\
\dot{S}_{l a b}=\alpha_{4} \bar{\rho}^{\gamma_{1}} \dot{\varepsilon}_{p}-c_{2} S_{l a b} \dot{\bar{\theta}}_{\text {sub }}-\alpha_{3} \bar{\rho}^{\gamma_{l a b}} S_{l a b}^{2} \bar{\theta}_{\text {sub }}^{\gamma_{\theta}}\left(1-\ln \left(\bar{\theta}_{\text {sub }}\right)\right)-\alpha_{5} S_{l a b} S_{H A B} \bar{\rho}^{\gamma_{H A B}} \\
\dot{\bar{\theta}}_{\text {sub }}=\alpha_{6} \bar{\rho}^{\gamma_{2}} \dot{\varepsilon}_{p} S_{\text {lab }}^{2} \bar{\theta}_{\text {sub }}\left(\theta_{r} \bar{\theta}_{\text {sub }}-1\right) \ln \left(n \cdot \bar{\theta}_{\text {sub }}\right) \\
\dot{\sigma}=E\left(\dot{\varepsilon}_{T}-\dot{\varepsilon}_{p}\right) \\
S=S_{H A B}+S_{l a b} \\
f_{H A B}=S_{H A B} / S
\end{array}\right.
$$

where $\alpha_{1}=c_{\alpha 1} \exp \left(-\frac{Q_{a}}{R T}\right), \alpha_{2}=C_{\alpha_{2}} \frac{1}{T} \exp \left(-K_{g} \cdot T-\frac{Q_{b}}{R T}\right), \alpha_{3}=\frac{C_{\alpha_{3}}}{T} \exp \left(-K_{g} \cdot T-\frac{Q_{b}}{R T}\right)$, $\alpha_{4}=C_{\alpha_{4}} \exp \left(-\frac{Q_{a}}{R T}\right), \alpha_{5}=C_{\alpha_{5}} \exp \left(-\frac{Q_{a}}{R T}\right), \alpha_{6}=C_{\alpha_{6}} \exp \left(-\frac{Q_{a}}{R T}\right), n=\theta_{c} / \theta_{m}, \theta_{r}=\theta_{c} / \theta_{\text {sat }}$.

Effects of both work hardening $(H)$ and grains size $\left(d=2 / S_{H A B}\right)$ on the viscoplastic strain rate are considered in the proposed model, where work hardening of the material is dependent on dislocation density.

The accumulation of dislocations results from plastic deformation $\left(\dot{\varepsilon}_{p}\right)$ and is reduced by dynamic recovery and recrystallisation. Grain boundary migration $\left(\alpha_{2} S \bar{\rho}^{\gamma \alpha}\right)$ induces annihilation of dislocations. The number of dislocations does not always increase with deformation. It can be limited by density saturation and is influenced by the area of HABs or grain size.

Accumulation of dislocations during deformation results in the formation of new LABs $\left(\alpha_{4} \bar{\rho}^{\gamma_{1}} \dot{\varepsilon}_{p}\right)$. Simultaneously, subgrain rotation $\left(c_{2} S_{l a b} \dot{\bar{\theta}}_{\text {sub }}\right)$, LABs migration $\left(\alpha_{3} \bar{\rho}^{\gamma_{l a b}} S_{\text {lab }}^{2}\right)$ and HABs migration ( $\alpha_{5} S_{l a b} S_{H A B} \bar{\rho}^{\gamma_{H A B}}$ ), contribute to deceasing the LABs area. These mechanisms lead to annihilation of dislocations from LABs and therefore area of LABs no longer keeps increasing, which addresses the first comment on the original GM model by 
McQueen and Kassner (2004). Migration velocity of LABs is misorientation angle dependent and can be expressed as $\bar{\theta}_{\text {sub }}^{\gamma_{\theta}}\left(1-\ln \left(\bar{\theta}_{\text {sub }}\right)\right)$.

HABs can be formed through subgrain rotation $\left(c_{2} S_{l a b} \dot{\bar{\theta}}_{\text {sub }}\right)$ and LABs migration $\left(\alpha_{3} \bar{\rho}^{\gamma_{l a b}} S_{l a b}^{2}\right)$, and may be annihilated by HABs migration $\left(\alpha_{2} \bar{\rho}^{\gamma_{H A B}} S_{H A B}^{2}\right)$. Simultaneously operating formation and elimination mechanisms for both LABs and HABs lead to steady-state values of $f_{H A B}$ which partly addresses the second comment on the original GM model by McQueen and Kassner (2004)

Misorientation of LABs changes with the absorption of dislocations during deformation $\left(\bar{\rho}^{\gamma_{2}} \dot{\varepsilon}_{p}\right)$, but the rate and degree of subgrain rotation are dependent on misorientation angle $\left(\bar{\theta}_{\text {sub }} \ln \left(n \cdot \bar{\theta}_{\text {sub }}\right)\right)$ and subgrain size $\left(S_{\text {lab }}^{2}\right)$. A factor $\left(\theta_{r} \bar{\theta}_{s u b}-1\right)$ is used in the present model to address the subgrain misorientation saturation which is often observed in experiments (McQueen and Kassner, 2004).

Without consideration of LABs migration, saturation of misorientation angle and fraction of HABs, and influence of grain size change on dislocation density, the proposed model may to some extent reduce to the GM model.

\section{Model application to hot deformation}

\subsection{Model application and validation for aluminium alloy AA5052}

Gourdet (1997) studied the deformation behaviour of AA5052 using hot torsion experiments up to a maximum strain of 1.2. Flow stress data under different testing conditions, at temperatures of $290,333,377$ and $420{ }^{\circ} \mathrm{C}$ and strain rates of $0.001,0.033,0.01$ and $0.1 \mathrm{~s}^{-1}$, were obtained. They are plotted with symbols in Fig. 2. Microstructures were examined with $\mathrm{SEM} / \mathrm{EBSD}$ and the measured grain sizes and fractions of HABs under different strains are listed in Table 1.

Experimental data from five different temperature and strain rate test conditions, were used to calibrate the model. They are plotted with hollow symbols in Fig. 2, i.e., under the conditions of $290{ }^{\circ} \mathrm{C} / 0.001 \mathrm{~s}^{-1}, 333{ }^{\circ} \mathrm{C} / 0.01 \mathrm{~s}^{-1}, 333{ }^{\circ} \mathrm{C} / 0.1 \mathrm{~s}^{-1}, 333{ }^{\circ} \mathrm{C} / 0.01 \mathrm{~s}^{-1}$ and $420{ }^{\circ} \mathrm{C} / 0.01 \mathrm{~s}^{-1}$. The evolution of grain size and fraction of HABs with strain, presented in Table 1, was also used for calibration. Two stress-strain curves respectively under $333{ }^{\circ} \mathrm{C} / 0.0033 \mathrm{~s}^{-1}$ and $377{ }^{\circ} \mathrm{C} / 0.01$ $\mathrm{s}^{-1}$ were used for validation. Material constants were determined against the experimental data using the techniques introduced by Lin and Yang (1999) and the determined constants are listed in Table 2. The determined constants were used to calculate evolution of both flow stress and microstructural variables employing MATLAB with an explicit integration method. Fig. 2 shows a comparison of stress-strain curves obtained from experiment and those calculated using the CDRX model under various deformation conditions. 

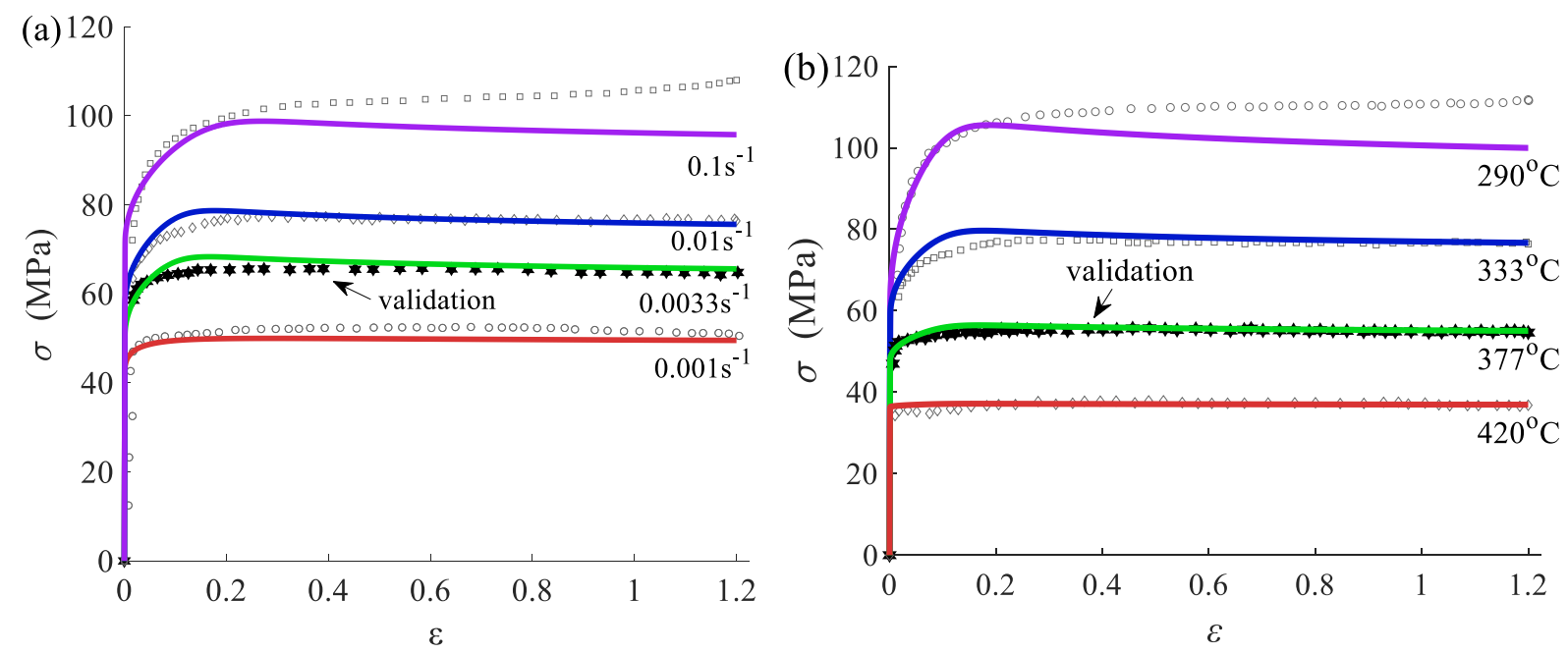

Fig. 2. Stress-strain curves for AA5052, symbols are experimental values, from Gourdet (1997), continuous lines from CDRX model. (a) Effect of strain rate at temperature $333^{\circ} \mathrm{C}$, (b) Effect of temperature at strain rate $0.01 \mathrm{~s}^{-1}$

Table 1 Evolution of fraction of HABs and grain size of AA5052 at different strains $\left(T=333{ }^{\circ} \mathrm{C}\right.$, $\dot{\varepsilon}=0.01 \mathrm{~s}^{-1}$ ) (Gourdet, 1997)

\begin{tabular}{lcccccc}
\hline Strain & 0 & 0.3 & 0.6 & 0.9 & 1.2 & 1.5 \\
\hline Grain Size $(\mu \mathrm{m})$ & 78 & 63 & 52 & 44 & 38 & - \\
$f_{H A B}$ & $87 \%$ & $20.7 \%$ & - & $23.4 \%$ & - & $32.8 \%$ \\
\hline
\end{tabular}

Table 2 Material constants for AA5052 alloy

\begin{tabular}{|c|c|c|c|c|c|c|c|c|}
\hline $\begin{array}{c}\boldsymbol{A}_{\mathbf{1}} \\
\left(\mathrm{s}^{-1}\right)\end{array}$ & $\begin{array}{c}\boldsymbol{A}_{2} \\
\left(\mathrm{MPa}^{-1}\right)\end{array}$ & $\begin{array}{c}\boldsymbol{B} \\
\left(\mathrm{MPa}^{-1}\right)\end{array}$ & $\begin{array}{c}\boldsymbol{k} \\
(\mathrm{MPa})\end{array}$ & $\begin{array}{l}c_{0} \\
(-)\end{array}$ & $\begin{array}{l}c_{1} \\
(-)\end{array}$ & $\begin{array}{l}c_{2} \\
(-)\end{array}$ & $\begin{array}{l}\gamma_{1} \\
(-)\end{array}$ & $\begin{array}{l}\gamma_{2} \\
(-)\end{array}$ \\
\hline $9.1 \times 10^{-5}$ & 0.165 & 48.53 & 24.30 & 2.25 & 0.15 & 0.05 & 0.05 & 0.15 \\
\hline$\gamma_{s}(-)$ & $\gamma_{d}(-)$ & $\gamma_{\theta}(-)$ & $\gamma_{l a b}(-)$ & $\gamma_{H A B}(-)$ & $\gamma_{\alpha}(-)$ & $\boldsymbol{n}(-)$ & \multicolumn{2}{|c|}{$\boldsymbol{c}_{\boldsymbol{\alpha} \mathbf{1}}\left(\mathrm{s}^{-1}\right)$} \\
\hline 0.01 & 0.01 & 6.18 & 0.45 & 4.95 & 4.12 & 0.75 & \multicolumn{2}{|c|}{0.13} \\
\hline $\begin{array}{c}\boldsymbol{c}_{\boldsymbol{\alpha} 2} \\
\left(\mu \mathrm{m} \mathrm{K} \mathrm{s}^{-1}\right)\end{array}$ & \multicolumn{2}{|c|}{$\begin{array}{c}c_{\alpha 3} \\
\left(\mu \mathrm{m} \mathrm{K} \mathrm{s}^{-1}\right)\end{array}$} & $\begin{array}{c}c_{\alpha 4} \\
\left(\mu \mathrm{m}^{-1}\right)\end{array}$ & $\begin{array}{c}c_{\alpha 5} \\
\left(\mu \mathrm{m}^{-1}\right)\end{array}$ & $\begin{array}{c}c_{\alpha 6} \\
\left(\mu \mathrm{m}^{2}\right)\end{array}$ & $\begin{array}{c}\boldsymbol{Q}_{\boldsymbol{a}} \\
\left(\mathrm{J} \mathrm{mol}^{-1}\right)\end{array}$ & \multicolumn{2}{|c|}{$\begin{array}{c}\boldsymbol{Q}_{\boldsymbol{b}} \\
\left(\mathrm{J} \mathrm{mol}^{-1}\right)\end{array}$} \\
\hline $2.34 \times 10^{6}$ & \multicolumn{2}{|c|}{$1.18 \times 10^{5}$} & 8.47 & $.50 \times 10^{4}$ & $5.77 \times 10^{6}$ & 17750 & \multicolumn{2}{|c|}{13400} \\
\hline
\end{tabular}

Although there is some deviation at high strain under the lowest temperature $\left(\mathrm{T}=290{ }^{\circ} \mathrm{C}\right)$ and the highest strain rate $\left(\dot{\varepsilon}=0.1 \mathrm{~s}^{-1}\right)$, Fig. 2 shows that a good agreement exists between calculated and experimental values. The root mean squared errors (RMSE) of the flow stress predictions under the deformation conditions of $333{ }^{\circ} \mathrm{C} / 0.033 \mathrm{~s}^{-1}$ and $377{ }^{\circ} \mathrm{C} / 0.01 \mathrm{~s}^{-1}$ are respectively $2.48 \mathrm{MPa}$ and $1.10 \mathrm{MPa}$, while the R-Squared of the flow stress predictions under the above two deformation conditions are respectively 0.9658 and 0.9974 . The slight deviations at low temperature and high strain rate may be due to the refinement of second phase particles, which is caused by the markedly increased deformation force under these conditions and hinders the process of dynamic recrystallisation (Gholinia et al., 2002).

Comparisons between the calculated results and the experimental data for the evolution of 
HABs fraction and grain size with strain can be made from the graphs shown in Fig. 3 and Fig. 4, respectively. The experimental data of Gourdet (1997) are shown as red stars while the calculated curve under the corresponding condition is the solid line. Curves calculated for other conditions are presented as dashed lines. In general, good agreement is achieved between modelled and experimental results. The average deviations for grain size and $f_{H A B}$ are $17.3 \%$ and $18.2 \%$ respectively.
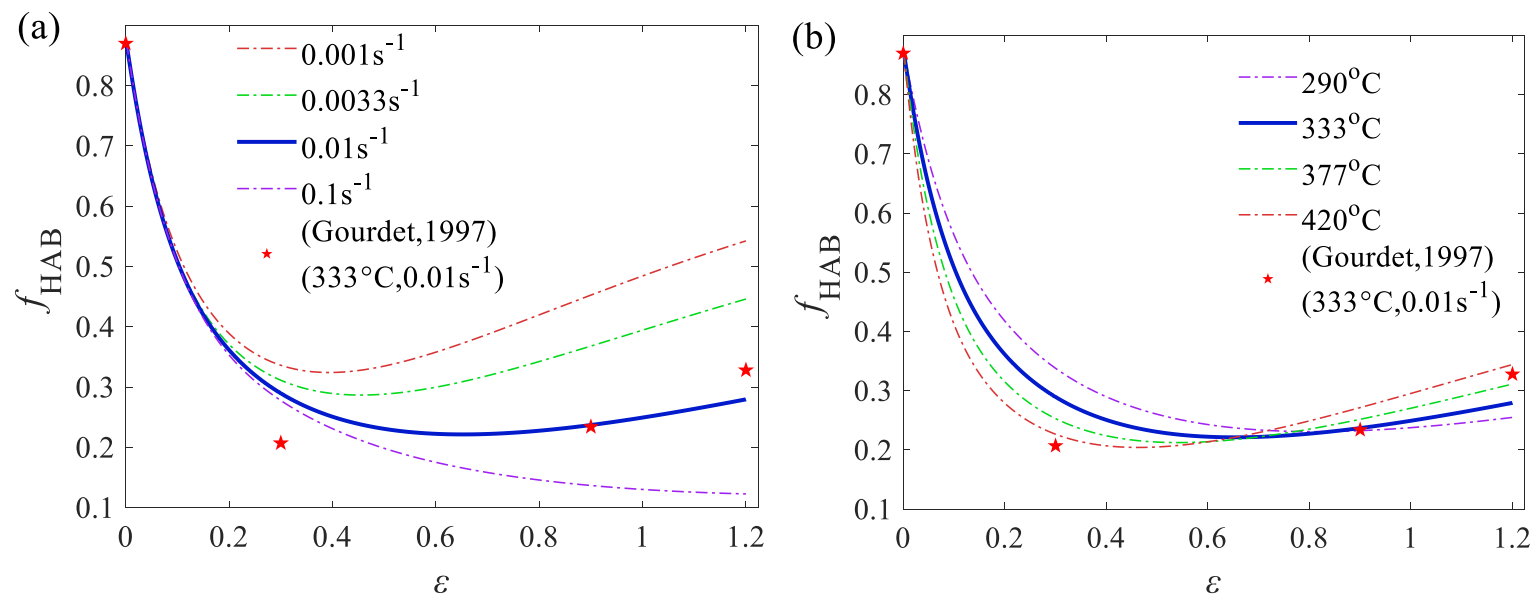

Fig. 3 Experimentally determined and calculated $f_{H A B}$, with respect to strain, for AA5052: (a) $T=$ $333{ }^{\circ} \mathrm{C}$; (b) $\dot{\varepsilon}=0.01 \mathrm{~s}^{-1}$
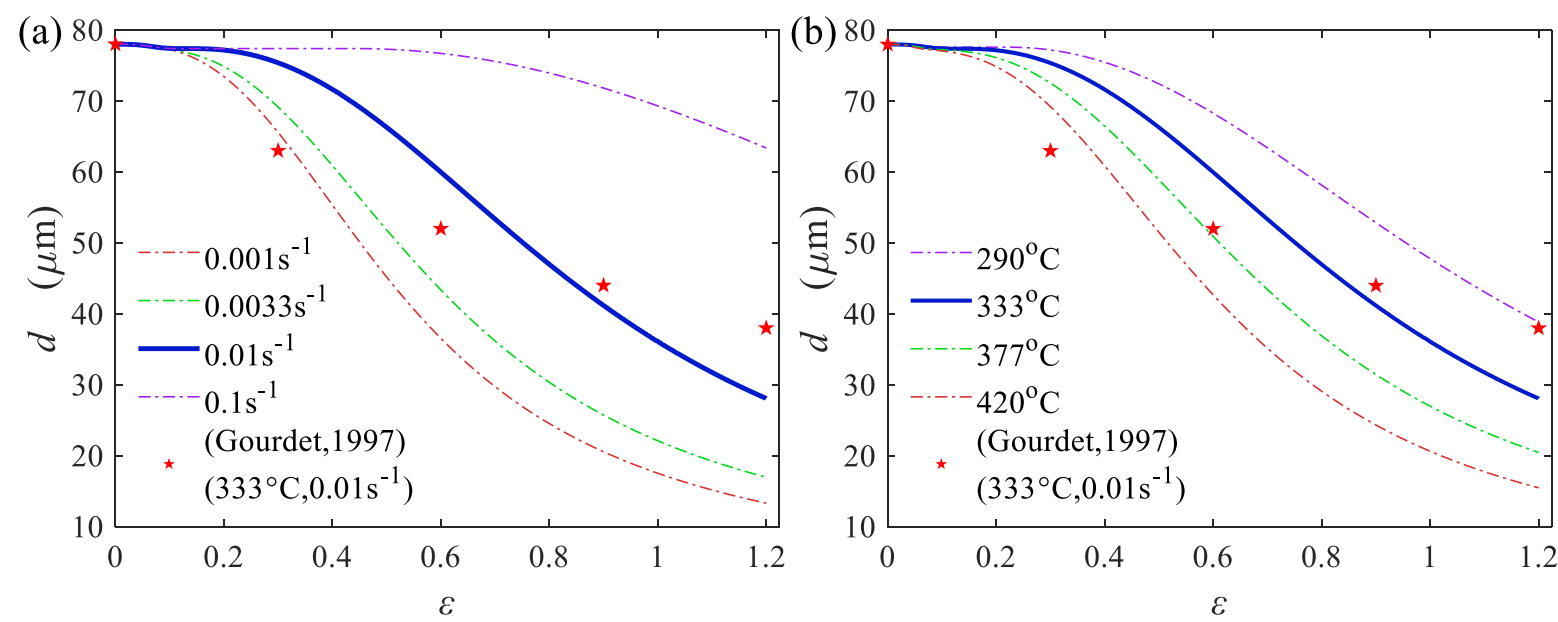

Fig. 4 Experimentally determined and calculated grain size, with respect to strain, for AA5052: (a) $T=$ $333{ }^{\circ} \mathrm{C}$; (b) $\dot{\varepsilon}=0.01 \mathrm{~s}^{-1}$

\subsection{Model application and validation for aluminium alloy AA7050}

AA7050 is widely used in the field of aerospace engineering because of its high strength-todensity ratio and excellent mechanical properties. It has been reported that dynamic recrystallisation of the AA7050-T7451 occurred at a deformation temperature of $400{ }^{\circ} \mathrm{C}$ and strain rate of $0.05 \mathrm{~s}^{-1}$ and progressed further with increasing temperature and decreasing strain rate (Li et al., 2019). Thus, this alloy was selected as a suitable material for validating the model.

Practical hot compression tests were carried out under various conditions (temperatures of $380,400,420$ and $440{ }^{\circ} \mathrm{C}$, and strain rates of $0.0005,0.005,0.05$ and $0.5 \mathrm{~s}^{-1}$ ), using a Gleeble- 
3800 machine. Cylindrical specimens with a diameter of $8 \mathrm{~mm}$ and height of $12 \mathrm{~mm}$ were annealed at $413{ }^{\circ} \mathrm{C}$ for 2 hours and then cooled in furnace to room temperature. For hot compression each specimen was heated at a rate of $2.5^{\circ} \mathrm{C} \mathrm{s}^{-1}$ to the target temperature and held for 3 minutes before compression. True stress-strain curves obtained from the tests are shown with symbols in Fig. 5.
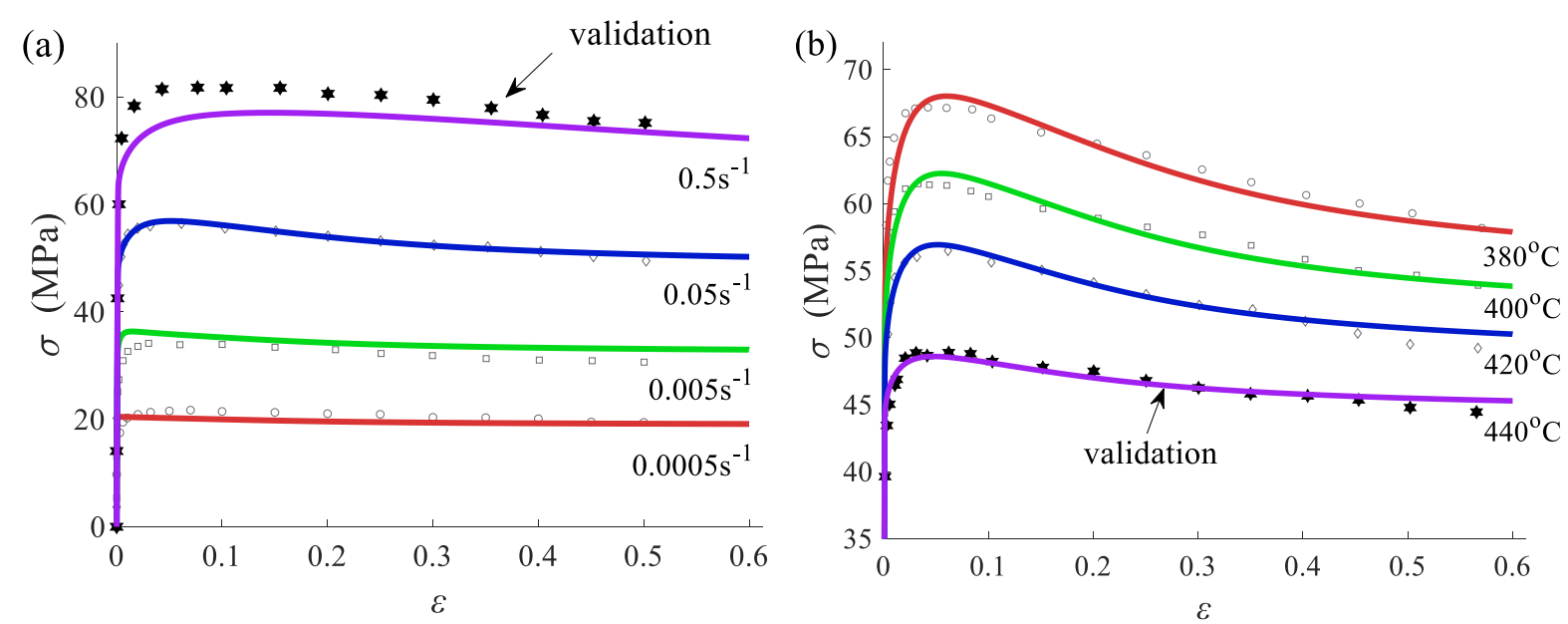

Fig. 5 True stress-strain curves for AA7050 from experiments (symbols) and modelling (lines), (a) $T=$ $420{ }^{\circ} \mathrm{C}$; (b) $\dot{\varepsilon}=0.05 \mathrm{~s}^{-1}$. Data with hollow symbols used for calibration, data with solid symbols used for validation.

Microstructural examination was carried out using SEM/EBSD to determine the evolution of areas of HABs and LABs. Initial area per unit volume and fraction of HABs of the annealed AA7050 alloys were $0.03502 \mu \mathrm{m}^{-1}$ and $43.8 \%$ respectively. Table 3 lists the measured $S_{H A B}$ and fractions of HABs under different strains.

Table 3 Measured values of $S_{H A B}$ and $f_{H A B}$ of AA7050-T7451 under different strains (deformed

\begin{tabular}{lccccc}
\multicolumn{7}{c}{ under $\left.T=420^{\circ} \mathrm{C}, \quad \dot{\varepsilon}=0.05 \mathrm{~s}^{-1}\right)$} \\
\hline Strain & 0 & 0.15 & 0.25 & 0.40 & 0.58 \\
\hline$S_{H A B}\left(\mu \mathrm{m}^{-1}\right)$ & 0.03502 & 0.04680 & 0.04826 & 0.05998 & 0.07699 \\
\hline$f_{H A B}(\%)$ & 43.8 & 50.9 & 54.3 & 53.7 & 53.0 \\
\hline
\end{tabular}

The experimental data obtained from deformation conditions, $380^{\circ} \mathrm{C} / 0.05 \mathrm{~s}^{-1}, 400{ }^{\circ} \mathrm{C} / 0.0005$ $\mathrm{s}^{-1}, 400{ }^{\circ} \mathrm{C} / 0.005 \mathrm{~s}^{-1}, 400{ }^{\circ} \mathrm{C} / 0.05 \mathrm{~s}^{-1}, 420{ }^{\circ} \mathrm{C} / 0.05 \mathrm{~s}^{-1}$, were used for calibration of the model and those under $400{ }^{\circ} \mathrm{C} / 0.5 \mathrm{~s}^{-1}, 440{ }^{\circ} \mathrm{C} / 0.05 \mathrm{~s}^{-1}$ were for validation. The determined material constants, used for modelling, are listed in Table 4. Fig. 5 indicates that the calculated flow stresses agree well with the experimental measurements. The root mean squared errors (RMSE) of the modelled flow stress under $\mathrm{T}=420{ }^{\circ} \mathrm{C}, \dot{\varepsilon}=0.5 \mathrm{~s}^{-1}$ and $\mathrm{T}=440^{\circ} \mathrm{C}, \dot{\varepsilon}=0.05 \mathrm{~s}^{-1}$ are 3.76 $\mathrm{MPa}$ and $1.43 \mathrm{MPa}$ respectively, while the R-Squared of the flow stress prediction under the same conditions are 0.9782 and 0.9983 respectively. Fig. 5 also indicates that dislocation density initially increases sharply with deformation, leading to the sharp increase of flow stress due to work hardening. As deformation progresses, dynamic recovery and recrystallisation occur, thus reducing dislocation density and work hardening. The flow stress therefore reaches 
a peak and then decreases as strain increases. Hence the model can replicate dynamic recovery and recrystallisation and can therefore predict the hardening and softening shown in flow stresses.

Table 4 Determined material constants for AA7050-T7451

\begin{tabular}{|c|c|c|c|c|c|c|c|c|}
\hline $\begin{array}{c}A_{1} \\
\left(\mathrm{~s}^{-1}\right)\end{array}$ & $\begin{array}{c}A_{2} \\
\left(\mathrm{MPa}^{-1}\right)\end{array}$ & $\begin{array}{c}\boldsymbol{B} \\
\left(\mathrm{MPa}^{-1}\right)\end{array}$ & $\begin{array}{c}\boldsymbol{k} \\
(\mathrm{MPa})\end{array}$ & $\begin{array}{l}c_{0} \\
(-)\end{array}$ & $\begin{array}{l}c_{1} \\
(-)\end{array}$ & $\begin{array}{l}c_{2} \\
(-)\end{array}$ & $\begin{array}{l}\gamma_{1} \\
(-)\end{array}$ & $\begin{array}{l}\gamma_{2} \\
(-)\end{array}$ \\
\hline $8.2 \times 10^{-4}$ & 0.148 & 18.50 & 13.80 & 15.00 & 1.15 & 0.70 & 0.70 & 0.15 \\
\hline$\gamma_{s}(-)$ & $\gamma_{d}(-)$ & $\gamma_{\theta}(-)$ & $\gamma_{l a b}(-)$ & $\gamma_{H A B}(-)$ & $\gamma_{\alpha}(-)$ & $\boldsymbol{n}(-)$ & \multicolumn{2}{|c|}{$\boldsymbol{c}_{\boldsymbol{\alpha} \mathbf{1}}\left(\mathrm{s}^{-1}\right)$} \\
\hline 5.00 & 0.01 & 6.18 & 0.77 & 2.10 & 4.20 & 0.75 & \multicolumn{2}{|c|}{38.11} \\
\hline $\begin{array}{c}\boldsymbol{c}_{\boldsymbol{\alpha} 2} \\
\left(\mu \mathrm{m} \mathrm{K} \mathrm{\textrm {s } ^ { - 1 }}\right)\end{array}$ & $\begin{array}{r}c \\
(\mu \mathrm{m}\end{array}$ & & $\begin{array}{c}c_{\alpha 4} \\
\left(\mu \mathrm{m}^{-1}\right)\end{array}$ & $\begin{array}{c}\boldsymbol{c}_{\boldsymbol{\alpha} \mathbf{5}} \\
\left(\mu \mathrm{m}^{-1}\right)\end{array}$ & $\begin{array}{c}c_{\alpha 6} \\
\left(\mu \mathrm{m}^{2}\right)\end{array}$ & $\begin{array}{c}\boldsymbol{Q}_{\boldsymbol{a}} \\
\left(\mathrm{J} \mathrm{mol}^{-1}\right)\end{array}$ & \multicolumn{2}{|c|}{$\begin{array}{c}\boldsymbol{Q}_{\boldsymbol{b}} \\
\left(\mathrm{J} \mathrm{mol}^{-1}\right)\end{array}$} \\
\hline $2.92 \times 10^{2}$ & 1.46 & & 14.15 & $2.0 \times 10^{3}$ & $1.61 \times 10^{5}$ & 17750 & \multicolumn{2}{|c|}{13400} \\
\hline
\end{tabular}

EBSD maps of the AA7050 alloys deformed to different strains at $T=420{ }^{\circ} \mathrm{C}$ and $\dot{\varepsilon}=$ $0.05 \mathrm{~s}^{-1}$ are shown in Fig. 6. It shows that as deformation progresses, the grains become more elongated, grain size is reduced, and fine recrystallised grains are formed. Grain refinement is most prominent at the largest strain tested, as shown in Fig. 6(d). Distributions of HABs and LABs for the initial structure $(\varepsilon=0)$ and the deformed structure $(\varepsilon=0.58)$ are presented in Fig. 7. The latter contains many recrystallised grains as shown in the white ovals. It shows that $(i)$ many recrystallised grains are concentrated along the original grain boundaries, (ii) the recrystallised grain size is similar to that of the subgrains, and (iii) a large number of LABs remain after recrystallisation. These three features are characteristic of a dominant CDRX mechanism (Sun et al., 2018).

Fig. 8 and Fig. 9 respectively illustrate experimentally determined and calculated evolution of area per unit volume and fraction of HABs versus strain. The calculated results corresponding the experimental conditions are plotted in solid lines. The maximum error, minimum error and average deviation are, $8.5 \%, 1.5 \%, 5.4 \%$ for $S_{H A B}$, and 5.4\%, $1.2 \%, 3.2 \%$ for $f_{H A B}$.
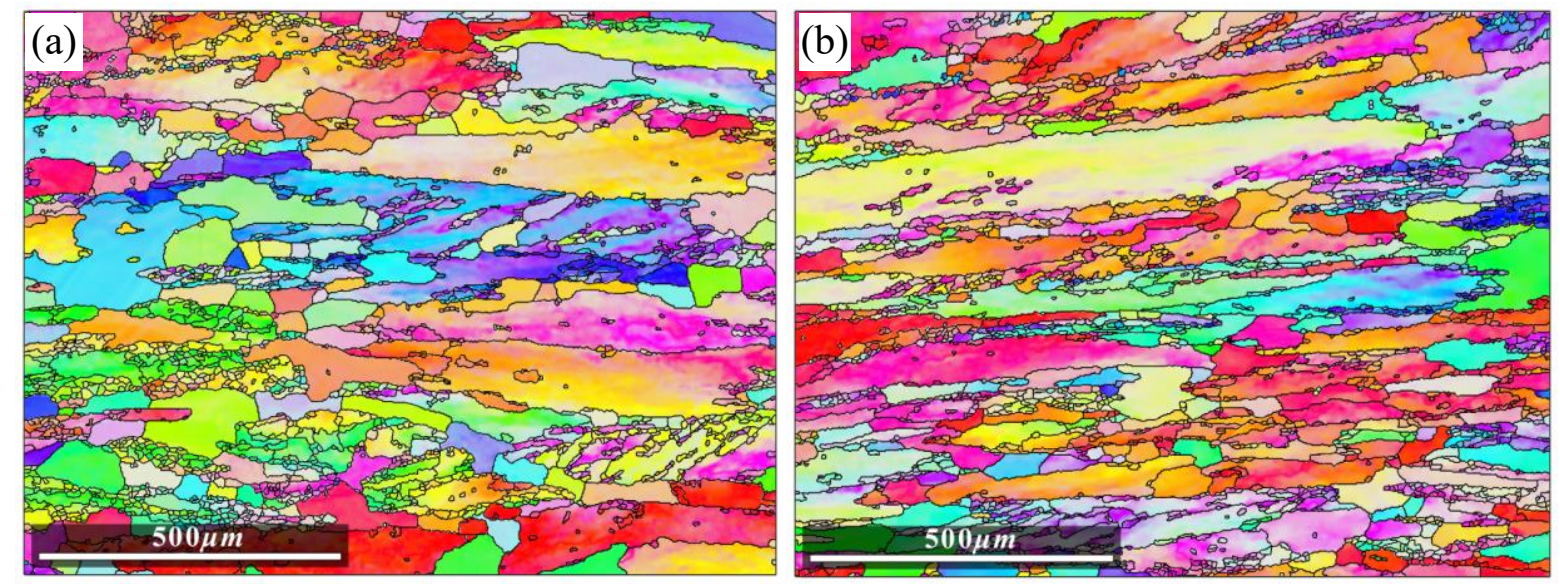

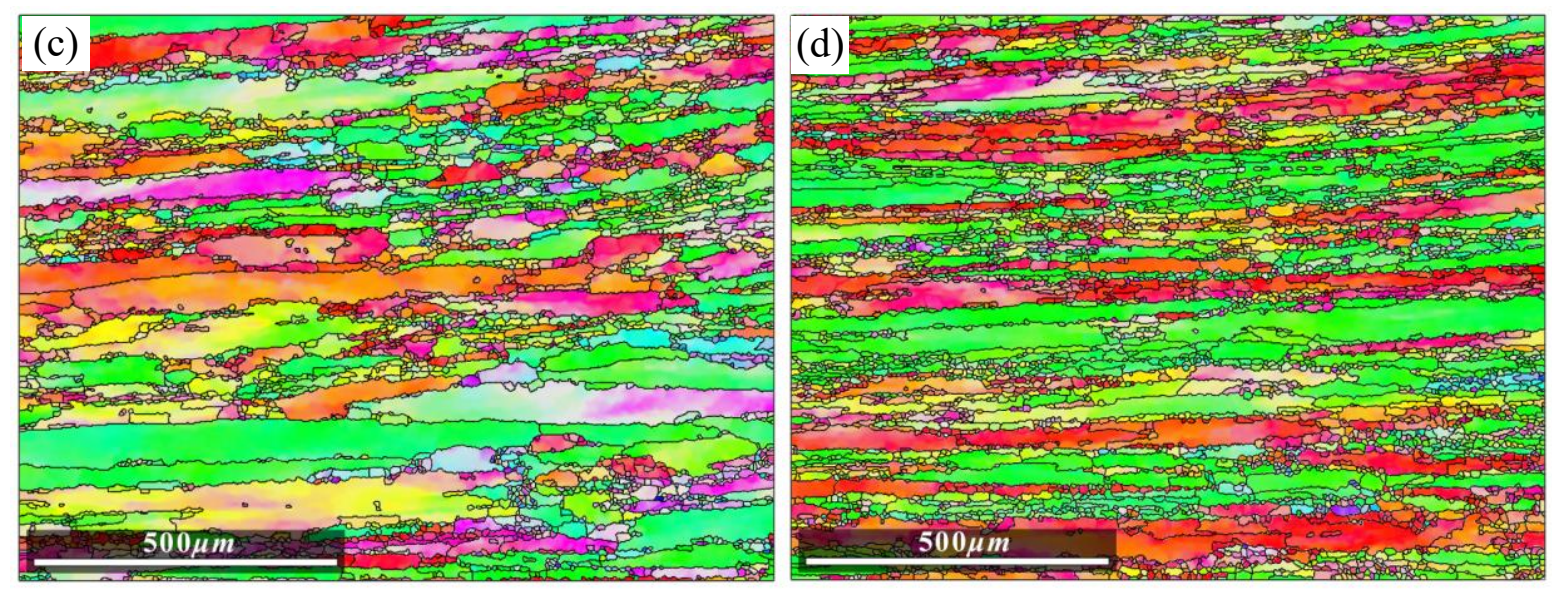

Fig. 6 EBSD maps of AA7050 deformed at $T=420{ }^{\circ} \mathrm{C}, \dot{\varepsilon}=0.05 \mathrm{~s}^{-1}$ : (a) $\varepsilon=0$ (initial structure); (b) $\varepsilon=0.15$; (c) $\varepsilon=0.4$; (d) $\varepsilon=0.58$.
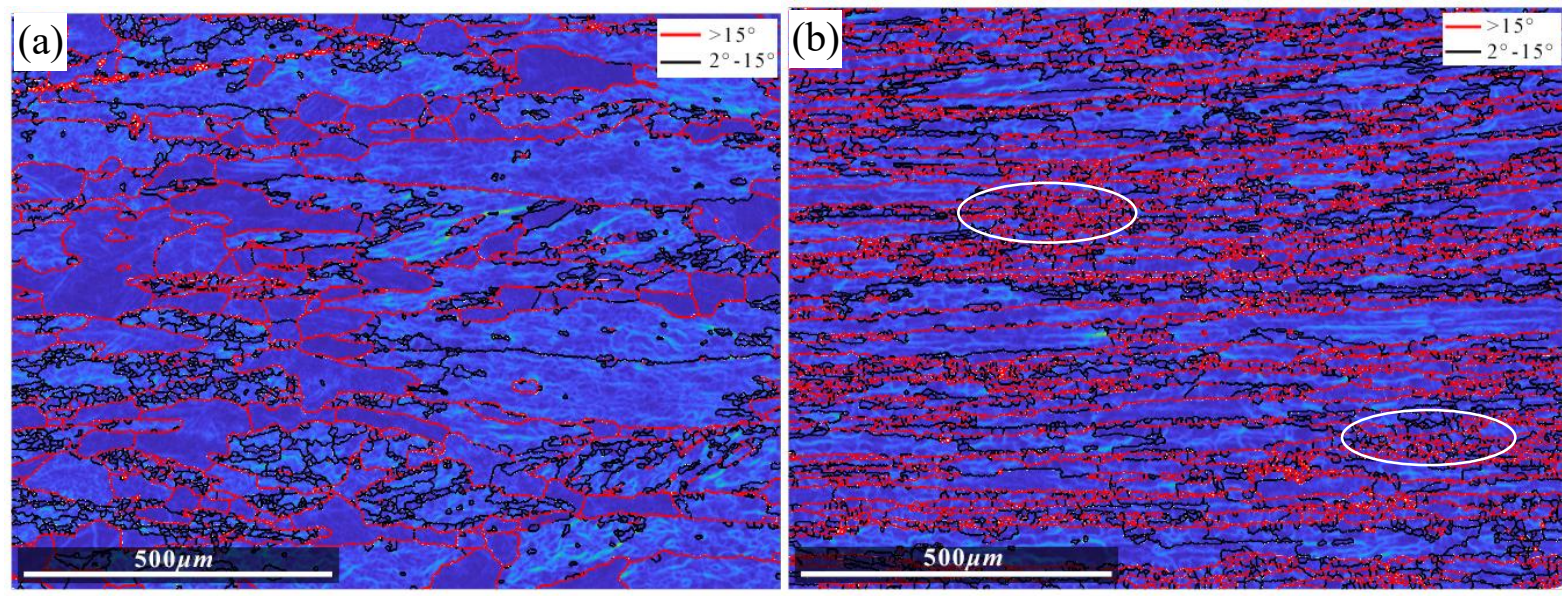

Fig. 7 Distribution of HABs (red lines) and LABs (black lines) of AA7050 deformed at $T=$ $420{ }^{\circ} \mathrm{C}, \dot{\varepsilon}=0.05 \mathrm{~s}^{-1}$ : (a) $\varepsilon=0$ (initial structure); (b) $\varepsilon=0.58$ (with many recrystallised grains, as shown in the white ovals).
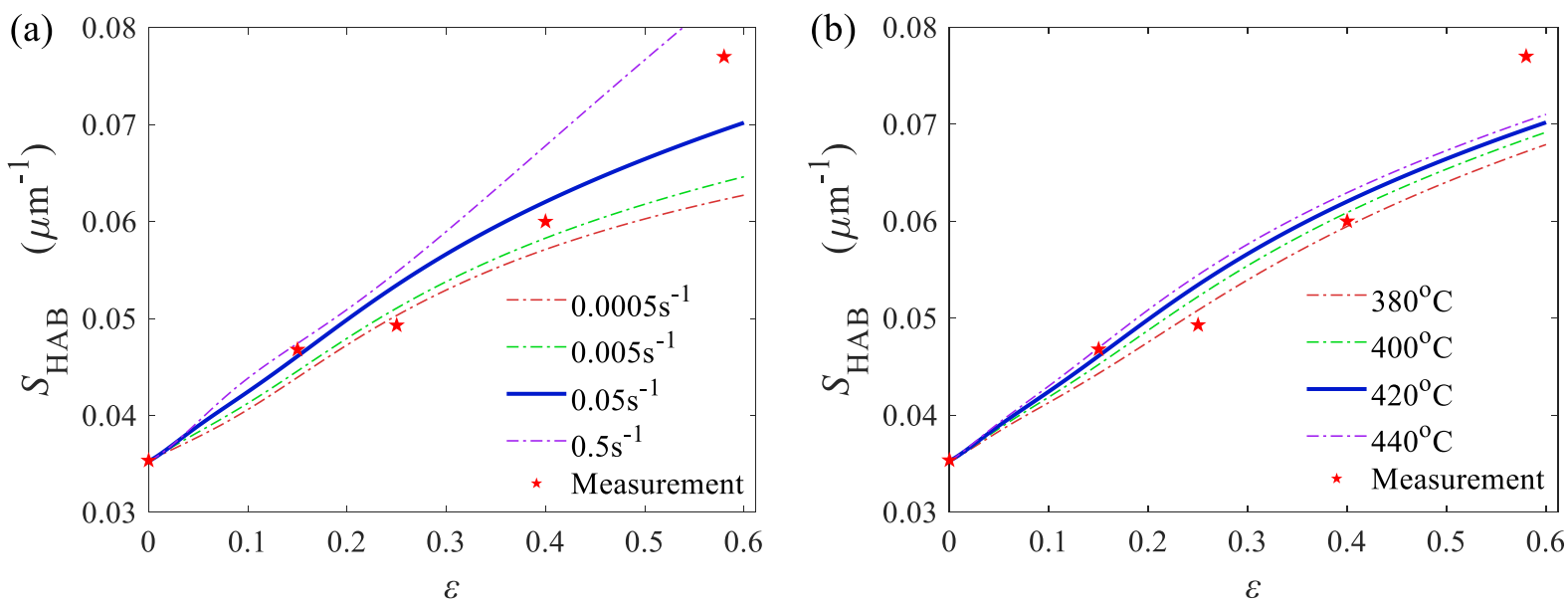

Fig. 8 HABs area per unit volume, calculated (lines), experimentally determined (symbols), for

$$
\text { AA7050: (a) } T=420{ }^{\circ} \mathrm{C} \text {; (b) } \dot{\varepsilon}=0.05 \mathrm{~s}^{-1}
$$



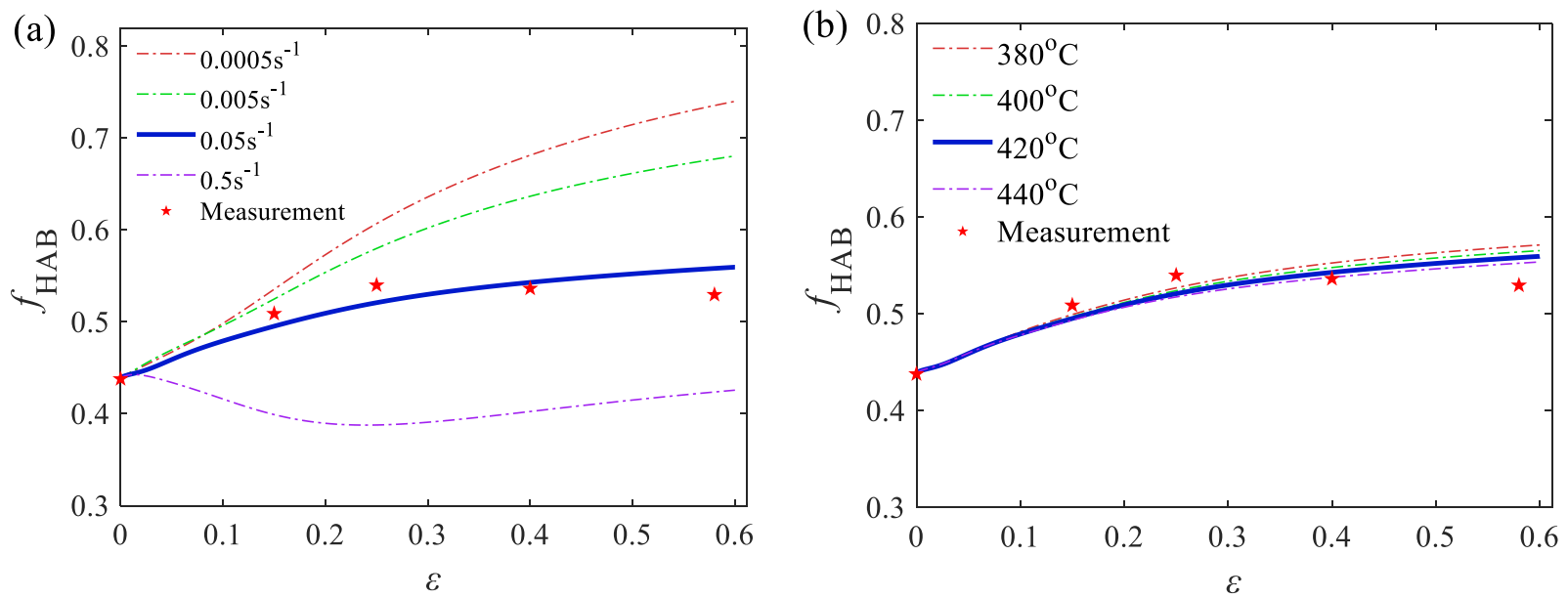

Fig. 9 Fraction of HABs, calculated (lines), experimentally determined (symbols), for AA7050: (a) $T$

$$
=420{ }^{\circ} \mathrm{C} \text {; (b) } \dot{\varepsilon}=0.05 \mathrm{~s}^{-1}
$$

\section{Discussion}

It can be seen from Fig. 3 that for AA5052 aluminium alloy, $f_{H A B}$ initially decreases with increasing strain. In general, at low strains, the HABs, even if mobile, will be prevented from migrating over large distances by the pinning pressures of the LABs with which they are in contact (Gholinia et al., 2002), leading to almost unchanged $S_{H A B}$ (and thus unchanged grain size $(d)$, as shown in Fig. 4; but $S_{l a b}$ keeps increasing at the low strain stage because of the formation of new LABs due to accumulation of dislocations, leading to a decreasing $f_{H A B}$. As strain continues to increase, misorientation of the LABs increases due to subgrain rotation and LABs migration and part of LABs gradually reaches the critical misorientation angle and changes to $\mathrm{HABs}$, resulting in the increase of $f_{H A B}$. However, spacing of HABs decreases with strain. When sufficient deformation is achieved, net boundary tensions will promote grain growth, driven by the reduction in total grain boundary area (Gholinia et al., 2002). Hence a dynamic balance between $S_{H A B}$ and $S_{l a b}$ will be reached, leading to the saturation of $f_{H A B}$.

The inhomogeneous distribution of second phase particles, such as $\mathrm{Al}_{3}(\mathrm{Sc}, \mathrm{Zr})$ dispersoids in AA7050 alloy, may contribute to boundary migration (Li et al., 2016) and lead to formation of additional high angle grain boundaries from an early stage (Gholinia et al., 2002), and therefore, make both $S_{H A B}$ and $f_{H A B}$ increase from the start of deformation (Fig. 8 and Fig. 9). Further experiments are required to examine the influence of second phase particles on the formation and elimination of $S_{H A B}$ and $S_{l a b}$.

From Fig. 3 and Fig. 9, it can be seen that both temperature and strain rate influence the $f_{H A B}$ values. Strain rate seems to have a much larger effect than temperature in the range investigated (3-4 orders of magnitude for strain rate and 60-120 degree for temperature). In fact, there is little effect on $f_{H A B}$ for AA7050 from 380 to $440{ }^{\circ} \mathrm{C}$ and small effect for AA5052 from 290 to $420^{\circ} \mathrm{C}$. For strain rate, the slower the deformation, the larger is the $f_{H A B}$. Dynamic recrystallisation is more likely to occur at higher temperatures and lower strain rates; more 
recrystallised grains with high angle boundaries are formed, leading to an increasing $S_{H A B}$, that is, a decreasing average grain size (Fig. 4 and Fig. 8). Such a finding is consistent with experimental results from other researchers, such as those of Xiao et al. (2018) and Zhao et al. (2018).

Many researchers observed that, when deformed to large strain $(\varepsilon=2-4)$, a saturation value of $f_{H A B}$ could be achieved for aluminium alloys. A wide range of saturation values have been reported for different alloys, e.g., 25\% 30\% for Al-Mg-Si alloys (Gourdet and Montheillet, 2000), 36\% for AA6061 (van Geertruyden et al., 2006), 60\% for Al-3Mg alloy (Gholinia et al., 2002). In some aluminium alloys, much higher fractions of HABs were observed, such as above $75 \%$ in the $\mathrm{Al}-\mathrm{Mg}-\mathrm{Si}$ alloy modified with $\mathrm{CaO}$-added $\mathrm{Mg}$ (Lee et al., 2016) and above $85 \%$ in the Al-Li-Mg-Sc alloys (Kaibyshev et al., 2005). This could be due to the higher dispersoid densities, which may stimulate the formation of micron sized grains with HABs and inhibit grain growth (Gholinia et al., 2002; Kaibyshev et al., 2005; Lee et al., 2016).

The model developed in this work can yield a stable $f_{H A B}$, between 0.4 and 0.9 under different deformation conditions, at a high strain of above 3 for both AA5052 and AA7050 alloys. It is consistent with the suggested saturation value of $f_{H A B}$ in the literature. However, experimental data of the saturated $f_{H A B}$ for both alloys are required to verify the model prediction accuracy under large strains (of above 3 ).

The saturation history of HABs under deformation, is not the same for different aluminium alloys. It is believed that the average misorientation angle of subgrains will be saturated to $4^{\circ} \sim 8^{\circ}$ (McQueen and Kassner, 2004) for aluminium alloys. Through observation and reasonable assumption of the average saturated misorientation angle of subgrains, different saturation processes have been modelled for different alloys and conditions, as shown in Fig. 10.

(a)

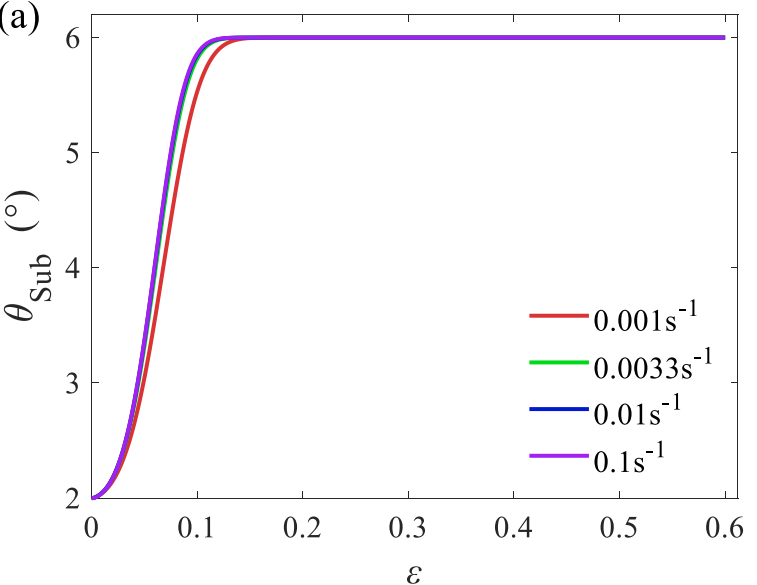

(b)

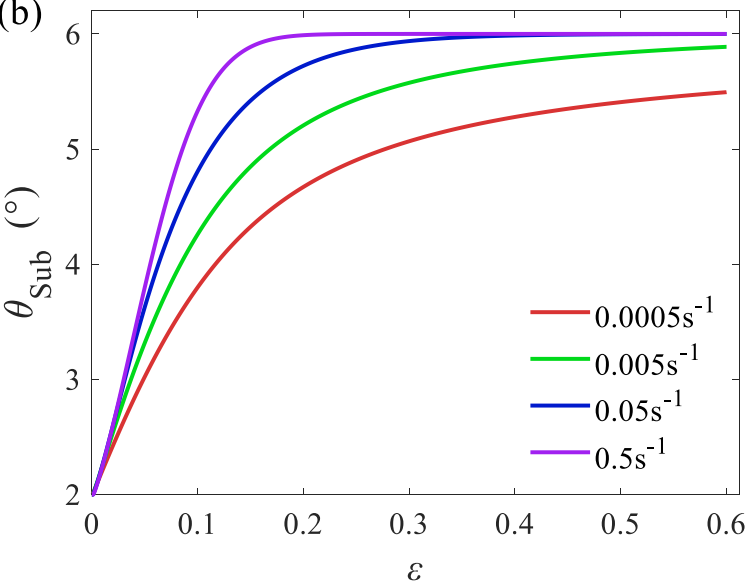

Fig. 10 Effect of strain rate on subgrain misorientation angle saturation process: (a) AA5052 at

$$
333^{\circ} \mathrm{C} \text {; (b) AA7050 at } 420^{\circ} \mathrm{C}
$$

It is shown in this study that reasonable results have been obtained for AA5052 and AA7050 from the proposed model. To further improve the model, some future investigations are suggested as follows:

(1) Experimental determination of steady-state grain size, saturated $f_{H A B}$ and average 
subgrain misorientation angle at large strains $(\varepsilon>3$, which can be achieved by torsion or equal channel angular pressing tests) for both alloys, to further validate and extend the application range of the model.

(2) Experimental determination of the effect of type, size and distribution of second phase particles and precipitates on the evolution of flow stress and microstructure and included in the constitutive equations of the model.

(3) Include other softening mechanisms, such as static recovery and static recrystallisation, in the model to make it usable for multi-pass hot forming processes with non-continuous deformation.

It should be emphasised that the present model is able to represent the deformation and evolution of inhomogeneous structures under hot forming conditions. All microstructural entities quantified by the model, e.g. misorientation angle $\left(\theta_{s u b}\right)$, area of HABs per unit volume $\left(S_{H A B}\right)$, area of LABs per unit volume $\left(S_{l a b}\right)$, grain size $(d)$ and subgrain size $(\delta)$ are average values. The model does not consider the effect of the actual crystallographic structure or orientation of any single generic crystal or grain and therefore, lacks the dependence on possible texture. However, for most alloys with isotropic properties, the effect of this deficiency is expected to be limited, and the model can be used to provide guidance on optimised processing to obtain required microstructure and properties.

\section{Conclusions}

A new set of unified constitutive equations, based on the continuous dynamic recrystallisation (CDRX) mechanism, was proposed in this study. Subgrain rotation, LABs and HABs migration contribute to the evolutions of the boundary areas of HABs and LABs, which directly influence the fraction of HABs $\left(f_{H A B}\right)$ and grain size $(d)$ or the area of HABs per unit volume, $\left(S_{H A B}\right)$. They are also the key softening mechanisms and affect viscoplastic stress during hot forming. From the outcomes of the work the following specific conclusions can be drawn:

1) Three elementary mechanisms during hot deformation, strain hardening, dynamic recovery, and dynamic recrystallisation are considered in the proposed model and their interactive effects have been modelled. Dynamic recrystallisation is regarded as a grain boundary area evolution process. Deformation leads to generation of new LABs. Subgrain rotation and LABs migration increase misorientation of LABs and transform part of LABs to HABs; while migration of HABs decreases areas of HABs and LABs. In contrast to the GM model, both subgrain rotation and migration of LABs are contributing to transformation of LABs to HABs, and both LABs and HABs are of mobility and can be annihilated by grain boundary migration.

2) Misorientation angle of LABs does not increase indefinitely and a saturate subgrain misorientation angle is included in the model. Mobility of LABs depends on misorientation 
angle and is saturated at a value at which they are turned to HABs. It is suggested that saturation values of HABs fraction and average subgrain misorientation be obtained, through large strain tests, to enable prediction of microstructure to be more accurate.

3) Application of the model to two aluminium alloys (AA5052 and AA7050) has served to verify the model. The modelled evolution of flow stress, fraction of HABs and grain size (or the area of HABs) is in good agreement with the experimental data.

\section{Acknowledgements}

The strong support from the Aviation Industry Corporation of China (AVIC) the First Aircraft Institute (FAI) for this funded research is much appreciated. The research was performed at the AVIC Centre for Structural Design and Manufacture at Imperial College London. The authors are also grateful for the support from China Scholarship Council, National Natural Science Foundation of China (51575535) and National Key Research and Development Program of China (2018YFB1106005). The authors would like to thank Professor Trevor A. Dean from University of Birmingham for helpful discussions.

\section{References}

Baxter, G.J., Furu, T., Zhu, Q., Whiteman, J.A., Sellars, C.M., 1999. The influence of transient strain-rate deformation conditions on the deformed microstructure of aluminium alloy $\mathrm{Al}-1 \% \mathrm{Mg}$. Acta Materialia 47 , 2367-2376.

Beucia, B., Queyreau, S., Kahloun, C., Chaubet, D., Franciosi, P., Bacroix, B., 2019. Plastic strain-induced grain boundary migration (SIBM) in pure aluminum: SEM in-situ and AFM examinations. International Journal of Plasticity 115, 29-55.

Blum, W., Schlögl, C., Meier, M., 1995. Subgrain formation and subgrain boundary migration in Al-5Mg during high temperature deformation in the range of class A behaviour in comparison with pure aluminium. Zeitschrift fur Metallkunde 86, 631-637.

Blum, W., Zhu, Q., Merkel, R., McQueen, H.J., 1996. Geometric dynamic recrystallization in hot torsion of Al-5Mg-0.6 Mn (AA5083). Materials Science and Engineering: A 205, 23-30.

Castro-Fernandez, F., Sellars, C., 1988. Static recrystallisation and recrystallisation during hot deformation of Al-1Mg-1Mn alloy. Materials science and technology 4, 621-627.

Chen, S.F., Li, D.Y., Zhang, S.H., Han, H.N., Lee, H.W., Lee, M.G., 2020. Modelling continuous dynamic recrystallization of aluminum alloys based on the polycrystal plasticity approach. International Journal of Plasticity, 102710

Dillamore, I., Smith, C., Watson, T., 1967. Oriented nucleation in the formation of annealing textures in iron. Metal Science Journal 1, 49-54.

Doherty, R., Szpunar, J., 1984. Kinetics of sub-grain coalescence-A reconsideration of the theory. Acta Metallurgica 32, 1789-1798.

Driver, J., 2018. The limitations of continuous dynamic recrystallization (CDRX) of aluminium alloys. 
Materials Letters 222, 135-137.

Duan, X., Sheppard, T., 2002. Simulation of substructural strengthening in hot flat rolling. Journal of materials processing technology 125, 179-187.

Fazan, B., Sherby, O.D., Dorn, J.E., 1954. Some observations on grain boundary shearing during creep. JOM 6, 919-922.

Fratini, L., Buffa, G., 2005. CDRX modelling in friction stir welding of aluminium alloys. International Journal of Machine Tools and Manufacture 45, 1188-1194.

Furu, T., Nes, E., 1996. Substructure evolution during different hot deformation processes of commercial non-heat treatable aluminium alloys. Materials Science and Engineering: A 214, 122-132.

Gholinia, A., Humphreys, F., Prangnell, P., 2002. Production of ultra-fine grain microstructures in Al-Mg alloys by coventional rolling. Acta materialia 50, 4461-4476.

Gourdet, S., 1997. Etude des mécanismes de recristallisation au cours de la déformation à chaud de l'aluminium. Ecole Nationale Supérieure des Mines de Saint-Etienne.

Gourdet, S., Montheillet, F., 2000. An experimental study of the recrystallization mechanism during hot deformation of aluminium. Materials Science and Engineering: A 283, 274-288.

Gourdet, S., Montheillet, F., 2003. A model of continuous dynamic recrystallization. Acta Materialia 51, 2685-2699.

Henshall, G., Kassner, M., McQueen, H., 1992. Dynamic restoration mechanisms in Al-5.8 At. Pct Mg deformed to large strains in the solute drag regime. Metallurgical Transactions A 23, 881-889.

Hu, P., Liu, Y., Zhu, Y., Ying, L., 2016. Crystal plasticity extended models based on thermal mechanism and damage functions: application to multiscale modeling of aluminum alloy tensile behavior. International Journal of Plasticity $86,1-25$.

Huang, Y., Humphreys, F., 2000. Subgrain growth and low angle boundary mobility in aluminium crystals of orientation $\{110\}<001$. Acta materialia 48, 2017-2030.

Humphreys, F.J., Hatherly, M., 2012. Recrystallization and related annealing phenomena. Elsevier.

Kaibyshev, R., Shipilova, K., Musin, F., Motohashi, Y., 2005. Continuous dynamic recrystallization in an Al-Li-Mg-Sc alloy during equal-channel angular extrusion. Materials Science and Engineering: A 396, 341351.

Kassner, M.E., McMahon, M.E., 1987. The dislocation microstructure of aluminum deformed to very large steady-state creep strains. Metallurgical and Materials Transactions A 18, 835-846.

Khan, A.S., Liu, H., 2012. Variable strain rate sensitivity in an aluminum alloy: Response and constitutive modeling, International Journal of Plasticity 36, 1-14.

Khan, A.S., Liu, J., Yoon, J.W., Nambori, R., 2015. Strain rate effect of high purity aluminum single crystals: Experiments and simulations. International Journal of Plasticity 67, 39-52.

Krasnikov, V.S., Mayer, A.E., Pogorelko, V.V., 2020. Prediction of the shear strength of aluminum with $\theta$ phase inclusions based on precipitate statistics, dislocation and molecular dynamics. International Journal of Plasticity, 102672.

Kreyca, J., Kozeschnik, E., 2018. Analysis of the Temperature and Strain-Rate Dependences of Strain Hardening. Metallurgical and Materials Transactions A 49, 18-21. 
Laasraoui, A., Jonas, J., 1991a. Prediction of steel flow stresses at high temperatures and strain rates. Metallurgical transactions A 22, 1545-1558.

Lee, J.-W., Son, K.-T., Jung, T.-K., Yoon, Y.-O., Kim, S.K., Choi, H.-J., Hyun, S.-K., 2016. Continuous dynamic recrystallization behavior and kinetics of $\mathrm{Al}-\mathrm{Mg}-\mathrm{Si}$ alloy modified with $\mathrm{CaO}$-added $\mathrm{Mg}$. Materials Science and Engineering: A 673, 648-659.

Li, D.-f., Zhang, D.-z., Liu, S.-d., Shan, Z.-j., Zhang, X.-m., Wang, Q., Han, S.-q., 2016. Dynamic recrystallization behavior of 7085 aluminum alloy during hot deformation. Transactions of Nonferrous Metals Society of China 26, 1491-1497.

Li, H., Lin, J., Dean, T.A., Wen S.W., Bannister, A.C., 2009. Modelling of mechanical property recovery for a pipeline steel in annealing process, International Journal of Plasticity 25, pp1049-1065.

Li, H., Sun, X., Yang, H., 2016. A three-dimensional cellular automata-crystal plasticity finite element model for predicting the multiscale interaction among heterogeneous deformation, DRX microstructural evolution and mechanical responses in titanium alloys. International Journal of Plasticity 87, 154-180.

Li, J.C.M., 1962. Possibility of subgrain rotation during recrystallization. Journal of Applied Physics 33, 2958-2965.

Li, W., Liu, Y., Jiang, S., Luan, Q., Li, Y., Gu, B., Shi, Z., 2019. A study of thermomechanical behaviour and grain size evolution of AA7050 under hot forging conditions. International Journal of Lightweight Materials and Manufacture 2, 31-39.

Li, Y., Shi, Z., Lin, J., Yang, Y.-L., Rong, Q., Huang, B.-M., Chung, T.-F., Tsao, C.-S., Yang J.-R, Balint, D.S., 2017. A unified constitutive model for asymmetric tension and compression creep-ageing behaviour of naturally aged Al-Cu-Li alloy. International Journal of Plasticity 89, 130-149.

Lin, J., Liu, Y., 2003. A set of unified constitutive equations for modelling microstructure evolution in hot deformation. Journal of Materials Processing Technology 143-144, 281-285.

Lin, J., Liu, Y., Farrugia, D.C.J., Zhou, M., 2005. Development of dislocation based-unified material model for simulating microstructure evolution in multipass hot rolling, Philosophical Magazine A 85, 1967-1987.

Lin, J., Yang, J., 1999. GA-based multiple objective optimisation for determining viscoplastic constitutive equations for superplastic alloys, International Journal of Plasticity 15, 1181-1196.

Lin, Y., Chen, X.-M., 2011. A critical review of experimental results and constitutive descriptions for metals and alloys in hot working. Materials \& Design 32, 1733-1759.

Lin, Y., Dong, W.-Y., Zhou, M., Wen, D.-X., Chen, D.-D., 2018. A unified constitutive model based on dislocation density for an $\mathrm{Al}-\mathrm{Zn}-\mathrm{Mg}-\mathrm{Cu}$ alloy at time-variant hot deformation conditions. Materials Science and Engineering A 718, 165-172.

Liu, P., Zheng, S., Chen, K., Wang, X., Yan, B., Zhang, P., Shi, S.-Q., 2019. Point defect sink strength of low-angle tilt grain boundaries: A phase field dislocation climb model. International Journal of Plasticity 119, 188-199.

Lobkovsky, A.E., Warren, J.A., 2001. Sharp interface limit of a phase-field model of crystal grains. Physical Review E 63, 051605.

Maizza, G., Pero, R., Richetta, M., Montanari, R., 2018. Continuous dynamic recrystallization (CDRX) model for aluminum alloys. Journal of materials science 53, 4563-4573. 
Majta, J., Madej, Ł., Svyetlichnyy, D.S., Perzyński, K., Kwiecień, M., Muszka, K., 2016. Modeling of the inhomogeneity of grain refinement during combined metal forming process by finite element and cellular automata methods. Materials Science and Engineering: A 671, 204-213.

Mayer, A.E., Ebel, A.A., Al-Sandoqachi, M.K., 2020. Plastic deformation at dynamic compaction of aluminum nanopowder: Molecular dynamics simulations and mechanical model. International Journal of Plasticity 124, 22-41.

McQueen, H.J., Kassner, M.E., 2004. Comments on 'a model of continuous dynamic recrystallization' proposed for aluminum. Scripta Materialia 51, 461-465.

Mishra, A., Kad, B.K., Gregori, F., Meyers, M.A., 2007. Microstructural evolution in copper subjected to severe plastic deformation: Experiments and analysis. Acta Materialia 55, 13-28.

Mohamed, M.S., Foster, A.D., Lin, J., Balint, D.S., Dean, T.A., 2012. Investigation of deformation and failure features in hot stamping of AA6082: Experimentation and modelling. International Journal of Machine Tools and Manufacture 53, 27-38.

Nagra, J.S., Brahme, A., Lévesque, J., Mishra, R., Lebensohn, R.A., Inal, K., 2020. A new micromechanics based full field numerical framework to simulate the effects of dynamic recrystallization on the formability of HCP metals. International Journal of Plasticity 125, 210-234.

Nayan, N., Mahesh, S., Prasad, M., Murthy, S., Samajdar, I., 2019. A phenomenological hardening model for an aluminium-lithium alloy. International Journal of Plasticity 118, 215-232.

Neumann, R., Böhlke, T., 2016. Hashin-Shtrikman type mean field model for the two-scale simulation of the thermomechanical processing of steel. International Journal of Plasticity 77, 1-29.

Pettersen, T., Holmedal, B., Nes, E., 2003. Microstructure development during hot deformation of aluminum to large strains. Metallurgical and Materials Transactions A 34, 2737-2744.

Poirier, J.P., Nicolas, A., 1975. Deformation-Induced Recrystallization Due to Progressive Misorientation of Subgrains, with Special Reference to Mantle Peridotites. The Journal of Geology 83, 707-720.

Read, W.T., Shockley, W., 1950. Dislocation models of crystal grain boundaries. Physical review 78, 275.

Rios, P.R., Siciliano Jr, F., Sandim, H.R.Z., Plaut, R.L., Padilha, A.F., 2005. Nucleation and growth during recrystallization. Materials Research 8, 225-238.

Rokni, M.R., Zarei-Hanzaki, A., Roostaei, AliA., Abedi, H.R., 2011. An investigation into the hot deformation characteristics of 7075 aluminum alloy. Mater. Design.32, 2339-2344.

Shi, C., Lai, J., Chen, X., 2014. Microstructural evolution and dynamic softening mechanisms of Al-Zn-Mg$\mathrm{Cu}$ alloy during hot compressive deformation. Materials 7, 244-264.

Sun, Z., Wu, H., Cao, J., Yin, Z., 2018. Modeling of continuous dynamic recrystallization of Al-Zn-Cu-Mg alloy during hot deformation based on the internal-state-variable (ISV) method. International Journal of Plasticity 106, 73-87.

Tang, T., Zhou, G., Li, Z., Li, D., Peng, L., Peng, Y., Wu, P., Wang, H., Lee, M.-G., 2019. A polycrystal plasticity based thermo-mechanical-dynamic recrystallization coupled modeling method and its application to light weight alloys. International Journal of Plasticity 116, 159-191.

Tu, X., Shahba, A., Shen, J., Ghosh, S., 2019. Microstructure and property based statistically equivalent RVEs for polycrystalline-polyphase aluminum alloys. International Journal of Plasticity 115, 268-292. 
Upmanyu, M., Srolovitz, D.J., Lobkovsky, A., Warren, J.A., Carter, W., 2006. Simultaneous grain boundary migration and grain rotation. Acta Materialia 54, 1707-1719.

Van Geertruyden, W.H., Misiolek, W.Z., Wang, P.T., 2006. Grain structure evolution in a 6061 aluminum alloy during hot torsion. Materials Science and Engineering: A 419, 105-114.

Verheyden, S., Pires Da Veiga, L., Deillon, L., Mortensen, A., 2019. The effect of size on the plastic deformation of annealed cast aluminium microwires. Scripta Materialia 161, 58-61.

Xiao, W., Wang, B., Wu, Y., Yang, X., 2018. Constitutive modeling of flow behavior and microstructure evolution of AA7075 in hot tensile deformation. Materials Science and Engineering: A 712, 704-713.

Zhao, J., Deng, Y., Tan, J., Zhang, J., 2018. Effect of strain rate on the recrystallization mechanism during isothermal compression in 7050 aluminum alloy. Materials Science and Engineering: A 734, 120-128.

Zheng, J.-H., Lin, J., Lee, J., Pan, R., Li, C., Davies, C.M., 2018. A novel constitutive model for multi-step stress relaxation ageing of a pre-strained 7xxx series alloy. International Journal of Plasticity 106, 31-47. 